\title{
Conductance anomalies and the extended Anderson model for nearly perfect quantum wires
}

\author{
T. Rejec ${ }^{1}$ and A. Ramšak ${ }^{1,2}$ \\ ${ }^{1}$ J. Stefan Institute, 1001 Ljubljana, Slovenia \\ 2 Faculty of Mathematics and Physics, \\ University of Ljubljana, 1001 Ljubljana, Slovenia \\ J.H. Jefferson \\ QinetiQ, Sensors and Electronic Division, \\ St. Andrews Road, Great Malvern, \\ Worcestershire WR14 3PS, England
}

(Dated: October 25, 2018)

\begin{abstract}
Anomalies near the conductance threshold of nearly perfect semiconductor quantum wires are explained in terms of singlet and triplet resonances of conduction electrons with a single weaklybound electron in the wire. This is shown to be a universal effect for a wide range of situations in which the effective single-electron confinement is weak. The robustness of this generic behavior is investigated numerically for a wide range of shapes and sizes of cylindrical wires with a bulge. The dependence on gate voltage, source-drain voltage and magnetic field is discussed within the framework of an extended Hubbard model. This model is mapped onto an extended Anderson model, which in the limit of low temperatures is expected to lead to Kondo resonance physics and pronounced many-body effects.

PACS numbers: 73.23.-b, 85.30.Vw, 73.23.Ad, 72.10.-d
\end{abstract}

\section{INTRODUCTION}

Semiconductor quantum wires can be fabricated with effective wire widths down to a few nanometers; for ex ample, by heteroepitaxial growth on ' $\mathrm{n}$ '-groove surfacest and ridges 3 , cleaved edge over-growth 3 , etched wires with gatingt, a d gated two-dimensional electron gas (2DEG) structures 6 . More recently, there has been considerable interest in carbon nanotubes for which the quantum wire cross section can approach atomic dimensions. Such structures have potential for opto-electronic applications, such as light-emitting diodes, low-threshold lasers, singleelectron devices and quantum information processing.

Conductance steps in various types of quantum point contacts and quantum wires were found more than a decade ago 5 . These first experiments are broadly consistent with a simple non-interacting picture However, there are certain anomalies, some of which are believed to be related to electron-electron interactions and appear to be spin-dependent. In particular, a structure is seen in the rising edge of the conductance curve, starting at around $0.7\left(2 e^{2} / h\right)$ and merging with the first conductance plateau with increasing energy 6 . This structure, already visible in the early experiments 5 , can survive to temperatures of a few degrees and also persists under increasing source-drain bias, even when the conductance plateau has disappeared. Under increasing in-plane magnetic field, the structure moves down, eventually merging with the $e^{2} / h$ conductance plateau at very high fields and is not a transmission effect through a ballistic channeld A structure is seen also in high quality quantum wires 10 . In some experiments, an anomaly is seen at lower energy with conductance around $\left.0.3\left(2 e^{2} / h\right)^{2} \cdot 1\right]$. This can also survive to a few degrees, though is less robust than the 0.7 anomaly and is more readily suppressed by a magnetic field2. Recently the anomaly was confirmed also in back-gated 22 , in shallow-ptched 13 point contacts and in a ballistic quantum wire 4 . At low temperatures the anomaly exhibits a puzzling similarity with Kondo resonance behavior 15 , as do thermopower measurements 16 .

Theoretical work has attempted to explain these observations in various ways, including conductance suppression in a $\mathrm{futtinger} \mathrm{liquid} \mathrm{with} \mathrm{repulsive} \mathrm{interaction}$ and disprder 17, local spin-polarized density-functional theory 18 and spin-polarized sub-band 19 . Near the conduction threshold, there is a 'Coulomb blockade' and we have shown that this gives rise to spin-dependent resenances, for wires of both rectangulan 22 and cylindrical21 cross-section, with related anomalies in thermoelectric transport coefficients22. A similar singlet-triplet scenario was presented in Ref. 23 and a phenomenological approach is presented in Ref. 24. Recent studies have investigated the 0.7 anomaly in quantum point contacts within the Hartree-Fock approximationes, spinfluctuation backscatteringe 26 and in the framework of the Anderson model with related Kondo resonance behaviore7.

In Refs. 20,21,22 we suggested that these anomalies are related to weakly bound states and resonant bound states within the wire. These would arise, for example, from a small fluctuation in thickness of the wire in some region giving rise to a weak bulge. If this bulge is very weak then only a single electron will be bound. We may thus regard this system as an 'open' quantum dot in which the bound electron inhibits the transport of conduction electrons via the Coulomb interaction. Near the conduction 
threshold, there will be a Coulomb blockade and we show below that this also gives rise to a resonance, analogows to that which occurs in the single-electron transistores. This is a generic effect arising from an electron bound in some region of the wire and such binding may arise from a number of sources, which we do not consider explicitly. For example, in addition to a weak thickness fluctuation, a smooth variation in confining potential due to remote gates, contacts and depletion regions could contribute to electron confinement along the wire or gated 2DEG. A significant contribution to the single-electron confinement could also arise from its electronic polarization of the lattice or image charge.

In this paper we extend our previous study of a particular geometry of the quantum wire with a comprehensive analysis of a wide range of shapes and sizes of wire in order to demonstrate the generic and wide applicability of the phenomena. We study in particular the threshold of the conductivity of nearly perfect wires for which a single electron is bound. We express the conductance in terms of the two-electron scattering matrix. In order to extend the exact two-electron analysis into the true many-electron domain, we construct an extended Anderson model and analyze the influence of the corresponding momentum dependent coupling matrix elements.

The model is introduced in the next section and the special case of a cylindrical GaAs wire is derived in Appendix A. In Section III a detailed analysis of the twoelectron problem is presented in which one electron is weakly bound in the wire, giving rise to spin-dependent scattering of the other. Exact singlet and triplet scattering states are computed near the conductance threshold. In Section IV we then show how the solutions of the scattering problem may be used to determine conductance by an extension of the Landauer-Büttiker formula. This gives excellent agreement with a number of experiments on different kinds of quantum wire. The effect of finite magnetic field on the anomalies is presented and it is shown how they are related to the spin-split steps in perfect quantum wires. In the last Section we also examine the dependence of the anomalies on asymmetry introduced by finite source-drain voltage and summarize. Additional appendices are devoted to technical details on the solution of the two-electron wave function in an external potential, and the Hartree-Fock analysis.

\section{BASIC MODEL}

In previous work20.2122, we have considered a straight quantum wire with a small fluctuation in thickness giving rise to a weak 'bulge'. The precise details of the bulge are largely unimportant for what follows, the main requirement being that the change in the width of the wire is sufficiently gradual that inter-channel mixing of the transverse modes is negligible and that only one electron may be bound in the bulge region. The latter is always the case for a weak symmetric bulge, which has at least one bound state which can only sustain one electron due to Coulomb repulsion. The problem reduces to electrons moving in an effective weak potential well if we confine ourselves (by choice of gate voltage) to the Fermi energies for which no more than one transverse mode is occupied, i.e. the conductance threshold and the first conductance step. A typical effective potential well for such a bulge is shown in Fig. 1. Such a potential well may arise in other ways, such as an actual potential fluctuation due to a nearby unscreened charged impurity, or even some selfconsistent effect due to the electrons themselves through electronic polarization and image charge in a remote gate. We shall not consider the possible cause of this weak potential further but emphasize that because it may arise in many ways, the weak potential well model is very general with widespread applicability.

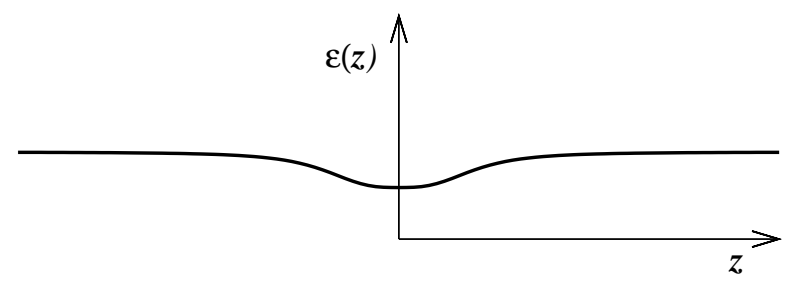

FIG. 1: Effective one-dimensional well caused by thickness fluctuation, impurity charge, gate image charge, selfpolarization due to single electron or some combination of these.

Consider now the motion of electrons in the wire near the conductance threshold. A single electron will be bound in the potential well region and the remaining electrons will undergo scattering from the localized electron via the Coulomb interaction as they propagate from source to drain. At sufficiently low Fermi energy, the electrons in the source contact will be totally reflected by the bound electron due to Coulomb repulsion and there will be no current from source to drain at $T=0$. As the Fermi energy is raised, the energy of the electrons in the source contact will be sufficiently high for them to overcome the Coulomb repulsion of the bound electron and a current will flow. In calculating this current we will make the approximation that the electrons flowing from source to drain only interact with the bound electron via a screened Coulomb interaction. This is a reasonable approximation provided that the electron density is not too low in the region of interest, i.e. the rising edge to the first conductance plateau. More precisely, the mean density of electrons in the wire (number per unit length) should be at least of order the inverse effective Bohr radius of the material. We return to this point again in the final section. Within this approximation, the manyelectron problem is reduced to an effective two-electron problem in which one electron is bound and the other is a representative electron at the Fermi energy in the leads. We show below that by solving this two-electron problem exactly and summing over all electrons near the Fermi energy we may compute the conductance. 


\section{A. Extended Hubbard and Anderson model}

The Hamiltonian corresponding to interacting electrons in the wire with a small geometric or potential inhomogeneity and close to the threshold of conduction is, within the effective-mass approximation an extended Hubbard Hamiltonian on a finite-difference lattice 29 ,

$$
H=\sum_{\sigma} H_{1 \sigma}+\frac{1}{2} \sum_{i \neq j} U_{i j} n_{i} n_{j}+\sum_{i} U_{i i} n_{i \uparrow} n_{i \downarrow} .
$$

Here $H_{1 \sigma}$ is the single-particle Hamiltonian:

$$
H_{1 \sigma}=-t \sum_{i}\left(c_{i+1 \sigma}^{\dagger} c_{i \sigma}+c_{i \sigma}^{\dagger} c_{i+1 \sigma}\right)+\sum_{i} \epsilon_{i} n_{i \sigma},
$$

where $c_{i \sigma}^{\dagger}, c_{i \sigma}$ are electron creation, annihilation operators, $n_{i \sigma}=c_{i \sigma}^{\dagger} c_{i \sigma}$, and $n_{i}=\sum_{\sigma} n_{i \sigma}$. Model parameters are hopping $t$, local potential at site $i, \epsilon_{i}$, and screened electron-electron interaction at sites $i$ and $j, U_{i j}$. This Hamiltonian is derived and justified in Appendix A.

In order to study the many-electron problem, it is also convenient to express the Hubbard Hamiltonian, Eq. (2.1), in a basis which distinguishes bound and unbound states explicitly. Single-electron solutions corresponding to the tight-binding Hamiltonian Eq. (2.2), follow from the single-particle Schrödinger equation

$$
H_{1}|\varphi\rangle=E_{1}|\varphi\rangle
$$

and (with omitted spin index $\sigma$ ), $|\varphi\rangle=\sum_{j} \varphi_{j} c_{j}^{\dagger}|0\rangle$. For large $|j|$ the potential $\epsilon_{j}$ is constant, therefore the solutions are asymptotically plane waves. We thus diagonalize this single-electron part of the Hamiltonian using the transformation $c_{q \sigma}^{\dagger}=\sum_{j} c_{j \sigma}^{\dagger} \phi_{j}^{q}$, where $\phi_{j}^{q}=$ $\langle j \mid q\rangle \sim \exp (i q j)$ asymptotically for unbound states, with eigenenergies $\varepsilon_{q}$. In this basis the Hamiltonian becomes,

$$
H=\sum_{q} \epsilon_{q} n_{q}+\frac{1}{2} \sum_{q_{1} q_{2} q_{3} q_{4} \sigma \sigma^{\prime}} \mathcal{U}\left(q_{1} q_{2} q_{3} q_{4}\right) c_{q_{1} \sigma}^{\dagger} c_{q_{3} \sigma^{\prime}}^{\dagger} c_{q_{4} \sigma^{\prime}} c_{q_{2} \sigma},
$$

where

$$
\mathcal{U}\left(q_{1} q_{2} q_{3} q_{4}\right)=\sum_{i j} U_{i j}\left(\phi_{i}^{q_{1}}\right)^{*} \phi_{i}^{q_{2}}\left(\phi_{j}^{q_{3}}\right)^{*} \phi_{j}^{q_{4}} .
$$

We further denote the lowest bound state with energy $\epsilon_{q}<0$ by $d_{\sigma} \equiv c_{q \sigma}$, with $n_{d}=\sum_{\sigma} d_{\sigma}^{\dagger} d_{\sigma}$ and, similarly, the scattering states with positive $\epsilon_{q}$ are distinguished by $q \rightarrow k$. There are two independent unbound states corresponding to each $k$ and these are chosen to be plane waves asymptotically, i.e. $\phi_{j}^{k} \rightarrow e^{i k j}$ as $j \rightarrow \pm \infty$ and $\epsilon_{k}=\frac{\hbar^{2} k^{2}}{2 m^{*}}$. Retaining only those Coulomb matrix elements which involve both localized and scattered electrons, omitting all terms which would give rise to states in which the localized state is unoccupied, we arrive at an Anderson-type
Hamiltonian29.30,

$$
\begin{aligned}
H & =\sum_{k} \epsilon_{k} n_{k}+\epsilon_{d} n_{d}+\sum_{k \sigma}\left(V_{k} n_{d \bar{\sigma}} c_{k \sigma}^{\dagger} d_{\sigma}+\text { h.c. }\right)+(2.6) \\
& +U n_{d \uparrow} n_{d \downarrow}+\sum_{k k^{\prime} \sigma} M_{k k^{\prime}} n_{d} c_{k \sigma}^{\dagger} c_{k^{\prime} \sigma}+\sum_{k k^{\prime}} J_{k k^{\prime}} \mathbf{S}_{\mathbf{d}} \cdot \mathbf{s}_{\mathbf{k k ^ { \prime }}} \cdot
\end{aligned}
$$

Here $U=\mathcal{U}(d d d d)$ is the Hubbard repulsion, $V_{k}=$ $\mathcal{U}(d d d k)$ is mixing term, $M_{k k^{\prime}}=\mathcal{U}\left(d d k k^{\prime}\right)-\frac{1}{2} \mathcal{U}\left(d k k^{\prime} d\right)$ corresponds to scattering of electrons and the direct exchange coupling is $J_{k k^{\prime}}=2 \mathcal{U}\left(d k k^{\prime} d\right)$. Spin operators in Eq. (2.7) are defined as $\mathbf{S}_{\mathbf{d}}=\frac{1}{2} \sum_{\sigma \sigma^{\prime}} d_{\sigma}^{\dagger} \boldsymbol{\sigma}_{\sigma \sigma^{\prime}} d_{\sigma^{\prime}}$ and $\mathbf{s}_{\mathbf{k} k^{\prime}}=\frac{1}{2} \sum_{\sigma \sigma^{\prime}} c_{k \sigma}^{\dagger} \boldsymbol{\sigma}_{\sigma \sigma^{\prime}} c_{k^{\prime} \sigma^{\prime}}$, where the components of $\boldsymbol{\sigma}$ are the usual Pauli matrices. A similar model has been proposed recently in Ref. 27. Although the Hamiltonian Eq. (2.7), is similar to the usual Anderson Hamiltonian 30 , we stress the important difference that the $k d$ hybridization term above arises solely from the Coulomb interaction, whereas in the usual Anderson case it comes primarily from one-electron interactions. These have been completely eliminated above by solving the oneelectron problem exactly. The resulting hybridization term contains the factor $n_{d \bar{\sigma}}$, and hence disappears when the localized orbital is unoccupied. This reflects the fact that an effective double-barrier structure and resonant bound state occurs via Coulomb repulsion only because of the presence of a localized electron.

To be specific, we consider in this paper a cylindrically symmetric quantum wire with symmetry axis $z$ and lateral coordinates $r$ and $\varphi 22$. Such a geometry corresponds to narrow 'v'-groove $z$-dependent quantum wires investigated recently, e.g. in Ref. 11. The diameter of the wire is $a(z)$, with zero potential within the wire and constant $V_{0}>0$ outside, i.e.,

$$
V(r, z)=\left\{\begin{array}{l}
0, \quad r<\frac{1}{2} a(z) \\
V_{0}, \quad r>\frac{1}{2} a(z)
\end{array} .\right.
$$

For the wire width, two generic shapes are taken, shown in Fig. 目with

$$
\begin{aligned}
& a(z)= \begin{cases}a_{0}\left(1-\xi \sin ^{2} \pi \frac{z}{a_{1}}\right), & |z|<a_{1} \\
a_{0}, & |z|>a_{1}\end{cases} \\
& a(z)=\left\{\begin{array}{ll}
a_{0}\left(1+\xi \cos ^{2} \frac{\pi}{2} \frac{z}{a_{1}}\right), & |z|<a_{1} \\
a_{0}, & |z|>a_{1}
\end{array} .\right.
\end{aligned}
$$
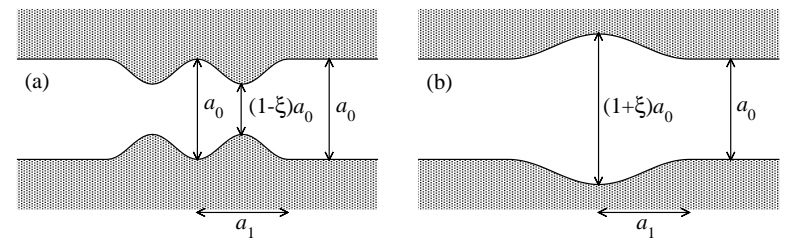

FIG. 2: The geometry of the 'open quantum dot' for the parameterization (a) Eq. 2.8a and (b) Eq. (2.8b). 
The region of interest is around $z=0$ and for large $|z|>a_{1}$ the diameter is constant $a_{0}$. Single particle solutions corresponding to this geometry as well as the derivation and calculation of parameters of the corresponding Hubbard Hamiltonian are presented in Appendix A.

\section{TWO-ELECTRON SOLUTIONS}

\section{A. Bound states}

In order to calculate conductance though the system we first solve the two interacting electron problem for the present geometry using the extended Hubbard Hamiltonian Eq. (2.1). Solutions for bound states are determined by numerical diagonalization of the system of equations presented in Appendix B, Eq. (B4). In Fig. 3 is shown the result of the two body electron density as a function of $z / a_{0}$ for various shapes of the bulge [Fig. 2(b)]. A general tendency is that long/narrow bulges correspond to stronger interaction resulting in formation of a double peak in density, as known from other studies of onedimensional quantum dots31. As long as the two peaks are not well separated, the approximate methods mentioned below are excellent, becoming gradually less reliable with increasing separation between the peaks.

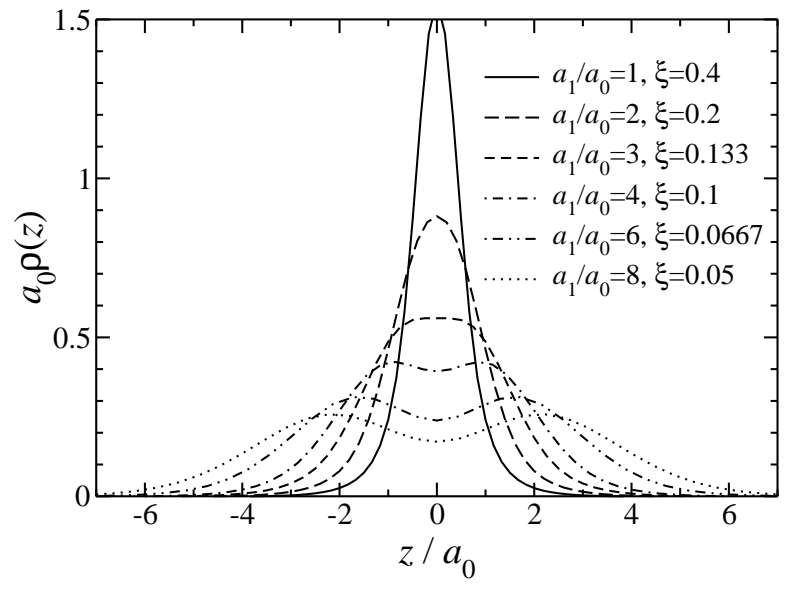

FIG. 3: Two-electron density for various bulge parameters.

In Fig. 4 we present typical examples of the energy of two bound (singlet) electrons $(E<0)$ where $\gamma$ is the electron-electron coupling strength, defined by replacement $U \rightarrow \gamma U$. Exact results are represented by the solid line, with other lines representing results obtained with the Hartree-Fock approximation, derived in Appendix C. At $E>0$ the lines correspond to the position of the singlet resonance, calculated with different methods and discussed below. In Fig. 5 the bulge is longer and narrower, therefore both singlet and triplet bound states exist for small $\gamma$, while for stronger coupling the triplet is first pushed into continuum and finally, for $\gamma \sim 0.7$, both states become resonances. Here approximate solutions are less accurate, because the bulge is much larger than in the previous case and therefore the problem is closer to the strong interaction limit, as is seen also in Fig. 3, dashed-dotted line, where the dip in the electron density signals the strong interaction regime. The Hartree-Fock approximation gives too large energies here, which are, however, qualitatively correct.

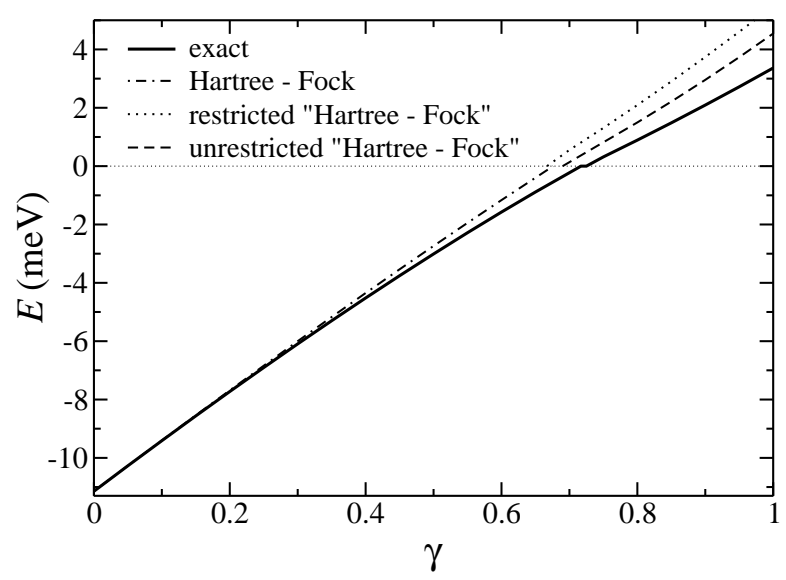

FIG. 4: Position of bound states $(E<0)$ and resonances $(E>0)$ vs. coupling $\gamma$, calculated exactly and within the Hartree-Fock approximation. Wire shape corresponds to Eq. (2.8b) with parameters: $a_{0}=a_{1}=10 \mathrm{~nm}, \xi=0.24$, $V_{0}=0.4 \mathrm{eV}$ and $\kappa=50 \mathrm{~nm}$.

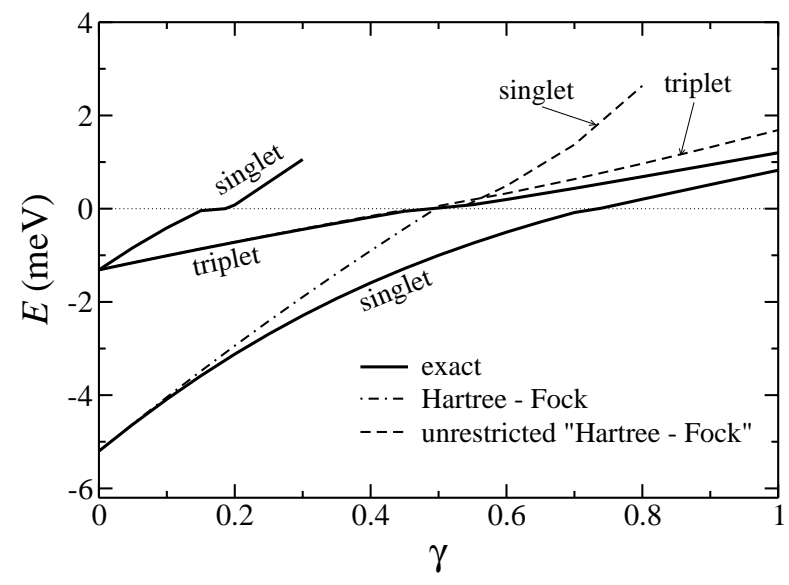

FIG. 5: As in Fig. 1 but with parameters: $a_{0}=10 \mathrm{~nm}$, $a_{1}=4 a_{0}, \xi=0.06, V_{0}=0.4 \mathrm{eV}$ and $\kappa=50 \mathrm{~nm}$.

\section{B. Scattering states}

Here we consider the scattering of an asymptotically free electron on a bound electron within the bulge. Such a system may be regarded as an "open quantum dot" in which one electron is bound and inhibits the transport of conduction electrons via Coulomb repulsion. The problem is analogous to treating the collision of an electron with a hydrogen atom as, e.g., described in Ref. 32 and 
studied by J.R. Oppenheimer and N.F. Mott33. We only consider here cases in which the energy of the scattered electron is smaller than the binding energy of the bound electron. This ensures that only elastic scattering is possible. Asymptotically the two-body wave function is a properly symmetrized product of a single particle bound state, $|\varphi\rangle$, and scattered state, $|\chi(E)\rangle$.

For two electrons, the antisymmetrized wavefunction can be written as a product of a spin-part and an orbital part. We write the orbital part as

$$
\widetilde{\psi}_{i j}=\frac{\psi_{i j}+(-1)^{S} \psi_{j i}}{\sqrt{2}},
$$

ensuring that this is symmetric for singlets $(S=0)$ and antisymmetric for triplets $(S=1)$ (see Appendix B). For some large $N \gg 1, \psi_{i j}$ takes the form

$$
\psi_{i j}=\left\{\begin{array}{ll}
\chi_{i}(E) \varphi_{j}+r^{(S)} \chi_{i}^{*}(E) \varphi_{j}, & i<-N \\
t^{(S)} \chi_{i}(E) \varphi_{j}, & i>N
\end{array} .\right.
$$

Asymptotic solutions for the unbound electron are obtained from the single-electron Hamiltonian Eq. (2.2) with the potential

$$
\tilde{\epsilon}_{j}=\epsilon_{j}+\sum_{k} U_{j k}\left|\varphi_{k}\right|^{2}
$$

for large $|j|$. Here $|\varphi\rangle$ is the single-particle bound state in the potential $\epsilon_{j}$. Solutions with forward and backward currents have the following asymptotic form $(j \rightarrow \infty)$

$$
\chi_{j}=\left\{\begin{array}{ll}
e^{ \pm i k j} & \kappa<\infty \\
e^{ \pm i(k j-\eta(k) \ln 2 k j)} & \kappa=\infty
\end{array},\right.
$$

for finite and infinite screening length $\kappa$, respectively (see Appendix A). With no screening $(\kappa=\infty) \chi_{j}$ are the Coulomb functions 34 .

Numerically exact solutions are obtained by solving a set of linear equations for the $(2 N+1)^{2}$ variables $\widetilde{\psi}_{i j}$ and transmission and reflection amplitudes.

\section{CONDUCTANCE}

\section{A. Single-electron solutions}

From the solution of the scattering problem, the conductance at zero temperature is alculated using the usual Landauer-Büttiker formalism 35 ,

$$
G=G_{0} \mathcal{T}(E),
$$

where $G_{0}=2 e^{2} / h, E$ is the Fermi energy (in this case $\left.E=E_{1}\right)$ and $\mathcal{T}(E)$ is the total transmission probability.

For an open bulge of shape Eq. (2.8a), Fig. 2(a), there are no bound states and only single-electron solutions 36 are relevant. In Fig. 6 we present $G$ as a function of electron energy for wires with shape Eq. (2.8a) and three different widths. The main effect is a change of energy scale, according to scaling rule Eq. (A7), and the magnitude of the conductance at the resonance energy, $G_{0}$. In Fig. 7 the conductance through the bulge of the shape from Eq. 2.8b) is presented. In contrast to the previous figure, a bound state can exist here, indicated by the dashed vertical line. Further lines $(n=1,2)$ indicate bound states of individual channels for the special case when channel mixing terms in Eq. (A4) are set to zero. Dips in the conductivity in the second plateau $\left(G \sim 3 G_{0}\right)$ correspond to Fano resonances caused by interchannel mixing terms.

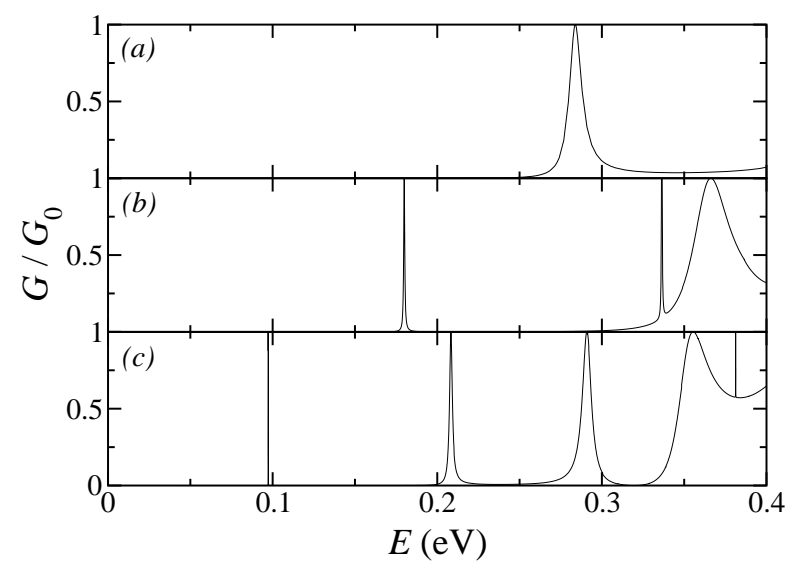

FIG. 6: $G$ for a wire with shape Eq. 2.8a and $V_{0}=0.4 \mathrm{eV}$, $\xi=0.8, a_{1}=a_{0}$ and in particular (a) $a_{0}=7 \mathrm{~nm}$, (b) $a_{0}=$ $10 \mathrm{~nm}$, and (c) $a_{0}=15 \mathrm{~nm}$.

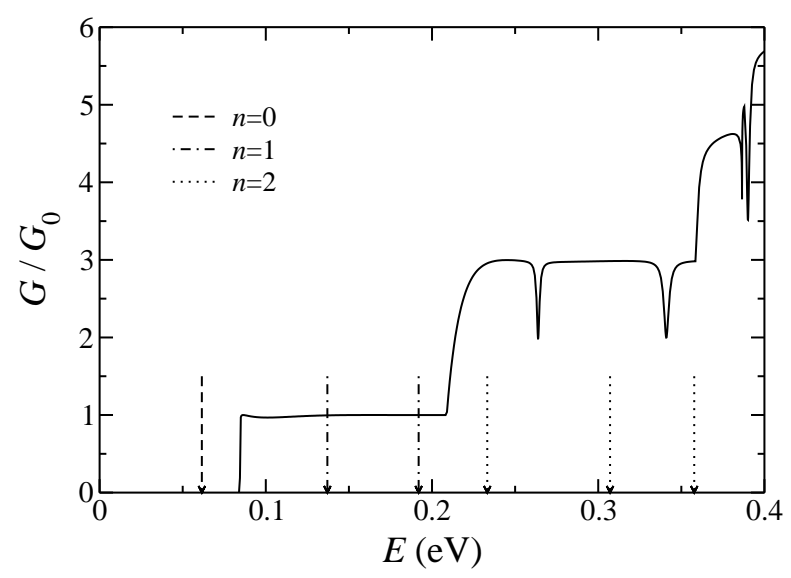

FIG. 7: $G$ for wire shape Eq. (2.8b) and $V_{0}=0.4 \mathrm{eV}, \xi=0.5$, $a_{0}=a_{1}=10 \mathrm{~nm}$. Vertical lines indicate positions of bound states for the lowest channels.

In Fig. 8(a) we again show the result of Fig. 7 comparing it with the one-channel approximation. In this paper we are interested in the rising edge of the conductance at the threshold, but with Coulomb interactions between bound and scattered electron included. Near threshold the one-channel approximation is excellent and therefore in the following we neglect higher channels. In Fig. 8(b) 
is presented the influence of discretization parameter $\Delta$, as introduced in Appendix A, on the conductivity. The position of the bound state is not strongly dependent on $\Delta$ (inset in enlarged energy scale) and for $\Delta<a_{1} / 5$ the results obtained on the lattice agree with the continuum calculation within a percent, which justifies the use of the discretized Hamiltonian, Eq. (2.1).

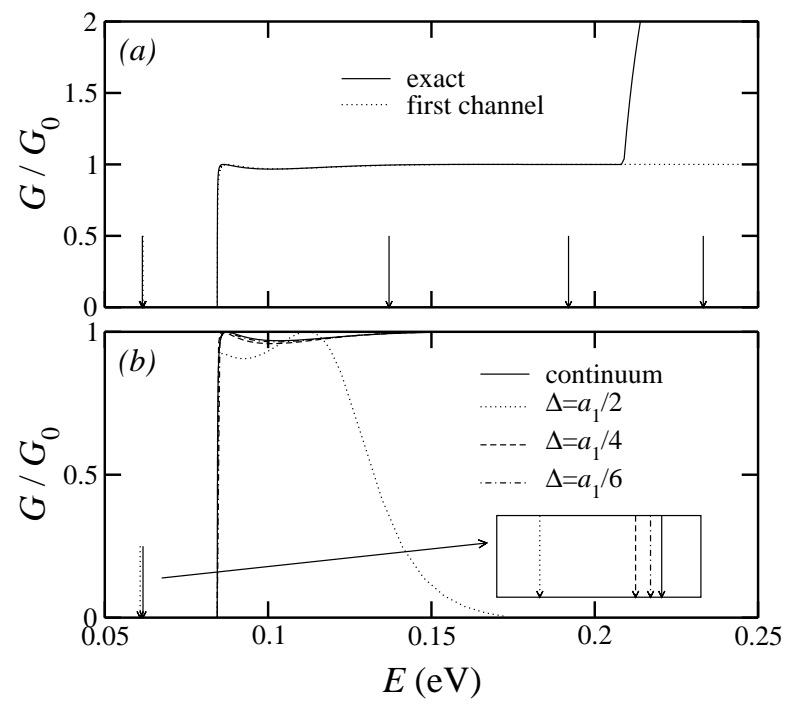

FIG. 8: (a) Conductance from Fig. 7 in comparison with the result obtained in the one channel approximation. (b) Conductance calculated with different $\Delta$ for wire parameters as in (a). Bound states are presented in the inset with enlarged energy scale.

\section{B. Interacting electrons}

We may extend the formula Eq. (4.1) to the case described in the previous section in which one electron is bound in the wire and the remaining electrons are transmitted with energy-dependent probability. Let $P_{\sigma}$ be the probability that the bound electron has spin $\sigma$. It follows directly that the conductance due to all spin-up electrons in the leads is given by the extended Landauer Büttiker formula:

$$
G_{\uparrow}=\frac{e^{2}}{h}\left[P_{\uparrow} \mathcal{T}_{\uparrow \uparrow}(E)+P_{\downarrow} \mathcal{T}_{\uparrow \downarrow}(E)\right],
$$

where $\mathcal{T}_{\uparrow \uparrow}$ is the transmission probability when the bound electron is spin up, $\mathcal{T}_{\uparrow \downarrow}$ is the transmission probability when the bound electron is spin down and $E$ is the Fermi energy. We have a similar expression for spin-down electrons in the leads and hence the total conductance is

$G(E)=\frac{e^{2}}{h}\left[P_{\uparrow} \mathcal{T}_{\uparrow \uparrow}(E)+P_{\downarrow} \mathcal{T}_{\uparrow \downarrow}(E)+P_{\uparrow} \mathcal{T}_{\downarrow \uparrow}(E)+P_{\downarrow} \mathcal{T}_{\downarrow \downarrow}(E)\right]$.

The transition probabilities $\mathcal{T}_{\uparrow \uparrow}$ and $\mathcal{T}_{\uparrow \downarrow}$ are different since in the former case the conduction and bound elec- trons both have the same spin (up) before and after scattering whereas in the latter case there are two possible final states, with or without spin-flip, i.e.

$$
\mathcal{T}_{\uparrow \downarrow}(E)=\left|t_{\uparrow \downarrow \rightarrow \uparrow \downarrow}\right|^{2}+\left|t_{\uparrow \downarrow \rightarrow \downarrow \uparrow}\right|^{2},
$$

where the scattering amplitudes are defined by

$$
\begin{aligned}
& \left\langle i \uparrow j \downarrow \mid \psi_{\uparrow \downarrow}\right\rangle \rightarrow t_{\uparrow \downarrow \rightarrow \uparrow \downarrow} \chi_{i} \varphi_{j} \\
& \left\langle i \downarrow j \uparrow \mid \psi_{\uparrow \downarrow}\right\rangle \rightarrow t_{\uparrow \downarrow \rightarrow \downarrow \uparrow} \chi_{i} \varphi_{j}
\end{aligned}
$$

as $i \rightarrow \infty .\left|\psi_{\uparrow \downarrow}\right\rangle$ is the exact scattering wavefunction and $\left|i \sigma j \sigma^{\prime}\right\rangle=c_{i \sigma}^{\dagger} c_{j \sigma^{\prime}}^{\dagger}|0\rangle . \varphi_{j}=\langle j \mid \varphi\rangle$ is the bound-state one-electron wavefunction and $\chi_{j}=\langle j \mid \chi\rangle$ is a forward propagating one-electron wavefunction at large $j$, as discussed in Section III.

In zero magnetic field it is clear that $P_{\uparrow}=P_{\downarrow}=\frac{1}{2}$ in Eq. (4.3). We can express $G(E)$ in a simpler form since the $\mathcal{T}_{\sigma \sigma^{\prime}}(E)$ are not all independent. Transforming to singlet and triplet base states (with $S_{z}=0$ ),

$$
\begin{aligned}
|s, i, j\rangle & =\frac{|i \uparrow, j \downarrow\rangle-|i \downarrow, j \uparrow\rangle}{\sqrt{2}}, \\
|t, i, j\rangle & =\frac{|i \uparrow, j \downarrow\rangle+|i \downarrow, j \uparrow\rangle}{\sqrt{2}},
\end{aligned}
$$

we get

$$
\begin{aligned}
\left\langle s, i, j \mid \psi_{\uparrow \downarrow}\right\rangle & \rightarrow \frac{t^{(0)}}{\sqrt{2}} \chi_{i} \varphi_{j} \\
\left\langle t, i, j \mid \psi_{\uparrow \downarrow}\right\rangle & \rightarrow \frac{t^{(1)}}{\sqrt{2}} \chi_{i} \varphi_{j},
\end{aligned}
$$

where

$$
\begin{aligned}
& t^{(0)}=t_{\uparrow \downarrow \rightarrow \uparrow \downarrow}+t_{\uparrow \downarrow \rightarrow \downarrow \uparrow} \\
& t^{(1)}=t_{\uparrow \downarrow \rightarrow \uparrow \downarrow}-t_{\uparrow \downarrow \rightarrow \downarrow \uparrow} .
\end{aligned}
$$

Hence, Eq. (4.2) becomes 32

$$
G=G_{0}\left(\frac{1}{4}\left|t^{(0)}\right|^{2}+\frac{3}{4}\left|t^{(1)}\right|^{2}\right) .
$$

\section{Results of numerical analysis}

In Fig. 9 we present the result of a comprehensive study of conductance for a variety of shapes of bulge. In Fig. 9(a) the bulge is wide and so short that only a singlet resonance is developed. The conductance therefore exhibits a structure similar to the 0.3 anomaly found in experiment37. In Figs. 9 (b,c) both singlet and triplet resonances are visible with a tendency for the resonances - to sharpen as the bulge becomes weaker $(\xi \rightarrow 0)$.

In Fig. 10 the wire width is fixed at $10 \mathrm{~nm}$ of the wire and positions of singlet (full lines) and triplet (dashed lines) resonances (or the corresponding bound states for $E<0)$ are plotted for various lengths and widths of 

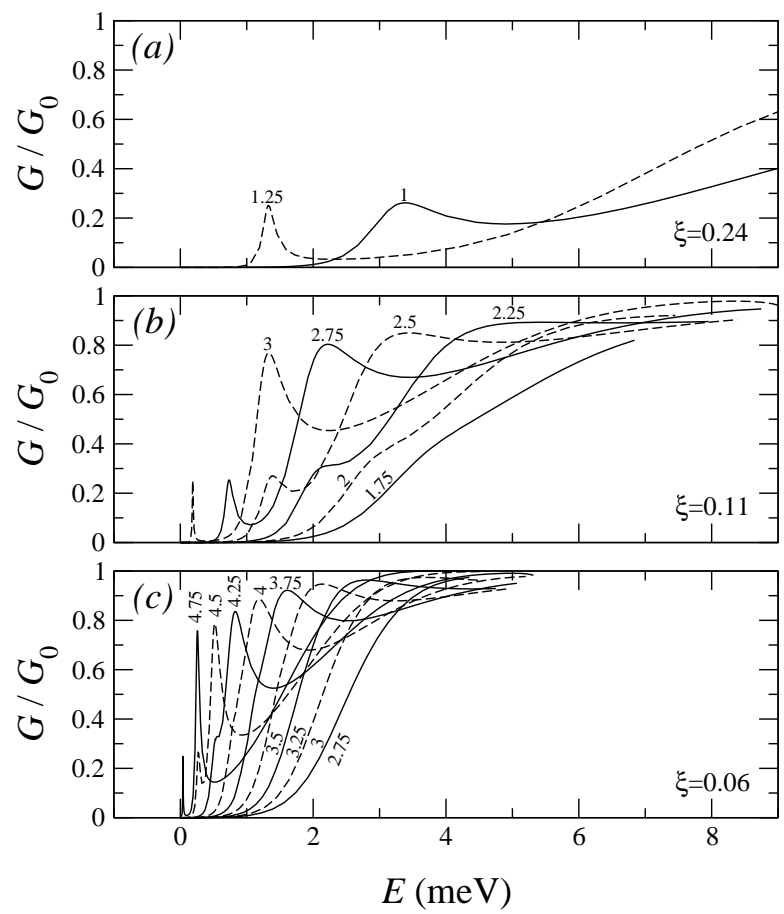

FIG. 9: Conductance for different shapes of the bulge. Each line is labeled with the parameter $a_{1} / a_{0}$. Other wire parameters: $a_{0}=10 \mathrm{~nm}, V_{0}=0.4 \mathrm{eV}$ and $\kappa=50 \mathrm{~nm}$.

bulge, represented by $a_{1} / a_{0}$ and $\xi$. We see that the resonances survive for a wide range of parameters. In Fig. 11 is shown the position of singlet and triplet resonance energies vs. the width of the wire, $a_{0}$, with fixed shape of the bulge. The insets show the energy dependence of singlet and triplet transmission probabilities for selected special cases. Note that we have scaled the energy by a factor $a_{0}^{2} E$. This would produce identical curves for non-interacting electrons [Eq. (A7)].

After performing calculations for a wide range of parameters, we conclude that a singlet resonance is always lower in energy than its corresponding triplet, in accordance with Lieb-Mattis theorem which however, is strictly valid only for ground states 3 .

\section{Magnetic field along the symmetry axis}

The nature of conductance anomalies studied here can be further illuminated with experiments done in a strong magnetic field 7 . The effect of the magnetic field is, in our treatment, taken into account via the usual Zeeman splitting of channel energies. The incoming electron with Fermi energy $E$ and spin component $S_{z}= \pm \frac{1}{2}$ then has kinetic energy

$$
E_{\mathrm{k}} \equiv E_{\mp}=E \mp E_{\mathrm{B}},
$$

where $E_{\mathrm{B}}=\frac{1}{2} g^{*} \mu_{\mathrm{B}} B . g^{*}$ is the effective gyromagnetic ratio and $\mu_{\mathrm{B}}$ is Bohr magneton.

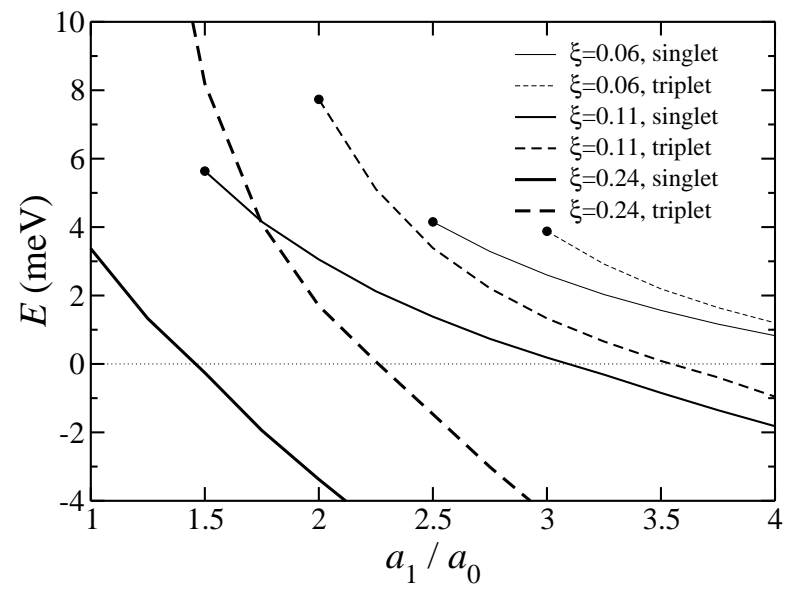

FIG. 10: Energies of singlet (full lines) and triplet (dashed lines) resonances and bound states for wire Eq. (2.8b) as a function of $a_{1} / a_{0}$ for different $\xi$. Other parameters are as in Fig. 9. Full circles represent the energy, where the resonance energy is above the 'ionization' energy and the underlying wave function form is not valid anymore.

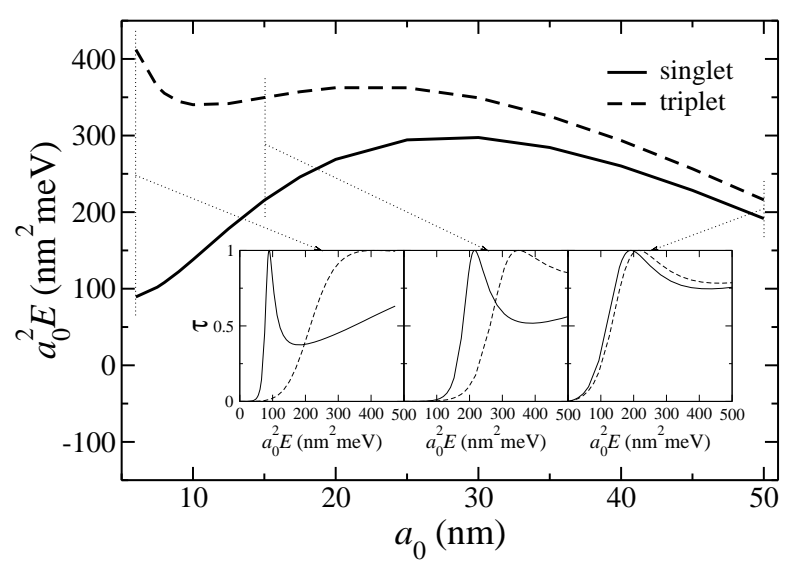

FIG. 11: The position of singlet (full line) and triplet (dashed line) resonances as a function of the width of the wire, $a_{0}$. Note that the energy of the resonances is presented in a scaled form. $a_{1}=50 \mathrm{~nm}, \xi=0.11$ and other parameters are as in Fig. 9 .

Near the conductance threshold we assume that the current is sufficiently low that the localized electron is in its ground-state with spin $\downarrow$ before each scattering event with a conduction electron. Hence $P_{\downarrow}=1$ and $P_{\uparrow}=0$ in Eq. (4.2) which becomes

$$
G_{\uparrow}(E, B)=\frac{e^{2}}{h} \mathcal{T}_{\uparrow \downarrow}(E, B) .
$$

In this case $G_{\downarrow} \neq G_{\uparrow}$ but, rather,

$$
G_{\downarrow}(E, B)=\frac{e^{2}}{h} \mathcal{T}_{\downarrow \downarrow}(E, B) .
$$

Since $\mathcal{T}_{\uparrow \downarrow}(E, B)=\mathcal{T}_{\uparrow \downarrow}\left(E_{-}, 0\right)$ and $\mathcal{T}_{\downarrow \downarrow}(E, B)=$ 
$\mathcal{T}_{\downarrow \downarrow}\left(E_{+}, 0\right)$, then the conductance is

$$
\begin{aligned}
G & =G_{\uparrow}+G_{\downarrow} \\
& =\frac{e^{2}}{h}\left[\mathcal{T}_{\uparrow \downarrow}\left(E_{-}, 0\right)+\mathcal{T}_{\downarrow \downarrow}\left(E_{+}, 0\right)\right] \\
& =\frac{e^{2}}{h}\left[\mathcal{T}_{t}\left(E_{+}, 0\right)+\frac{1}{2} \mathcal{T}_{t}\left(E_{-}, 0\right)+\frac{1}{2} \mathcal{T}_{s}\left(E_{-}, 0\right)\right],
\end{aligned}
$$

where $\mathcal{T}_{s}$ and $\mathcal{T}_{t}$ are the same functions as in zero magnetic field.

In Fig. 12(a) are plotted individual transmission probabilities for different spin configurations. Note that the spin-flip term is in general dominant at higher energies. In Fig. 12(b) the corresponding results for conductance in the presence of a magnetic field is shown. The full line corresponds to $B=0$, and other curves to $B$ in increments $\Delta B=10 \mathrm{~T}$.

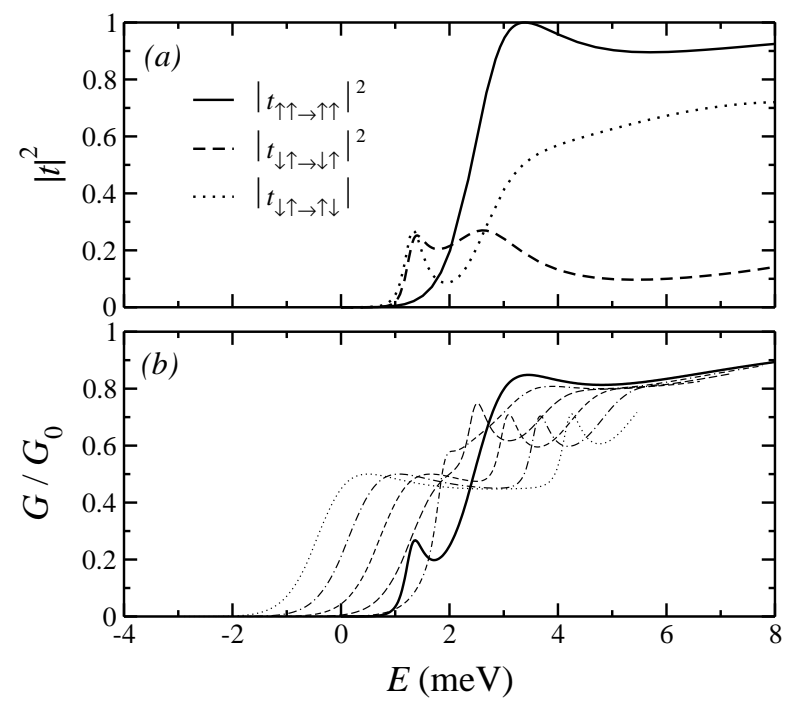

FIG. 12: (a) Transmission probabilities for relevant spin configurations. (b) Conductance for $B=0$ (full line) and other lines for $B$ in increments $\Delta B=10 \mathrm{~T}$. Parameters of the wire: $a_{0}=10 \mathrm{~nm}, a_{1}=2.5 a_{0}, \xi=1.11, V_{0}=0.4 \mathrm{eV}$ and $\kappa=50 \mathrm{~nm}$.

\section{E. Results for the Anderson model}

As shown in Section II, the Hubbard model studied above can be mapped onto an extended Anderson model, Eq. (2.7). Conductance through a quantum dot described by a standard Anderson model is basically described by a peak or several peaks and at higher energies the conductance approaches zero 39 . In the case of an open quantum dot, studied here, at higher energies the conductance tends toward unity, as a consequence of additional coupling parameters in the extended model. Here we analyze these terms individually and show their relative importance.

The coupling parameters are momentum dependent and in Fig. 13 the couplings $V_{k}, M_{k k^{\prime}}$ and $J_{k k^{\prime}}$ are shown.
Note that $M_{k k^{\prime}}$ at higher energies tends to a constant, while other parameters approach zero, ensuring the correct behavior at high-energy with unit transmission. The
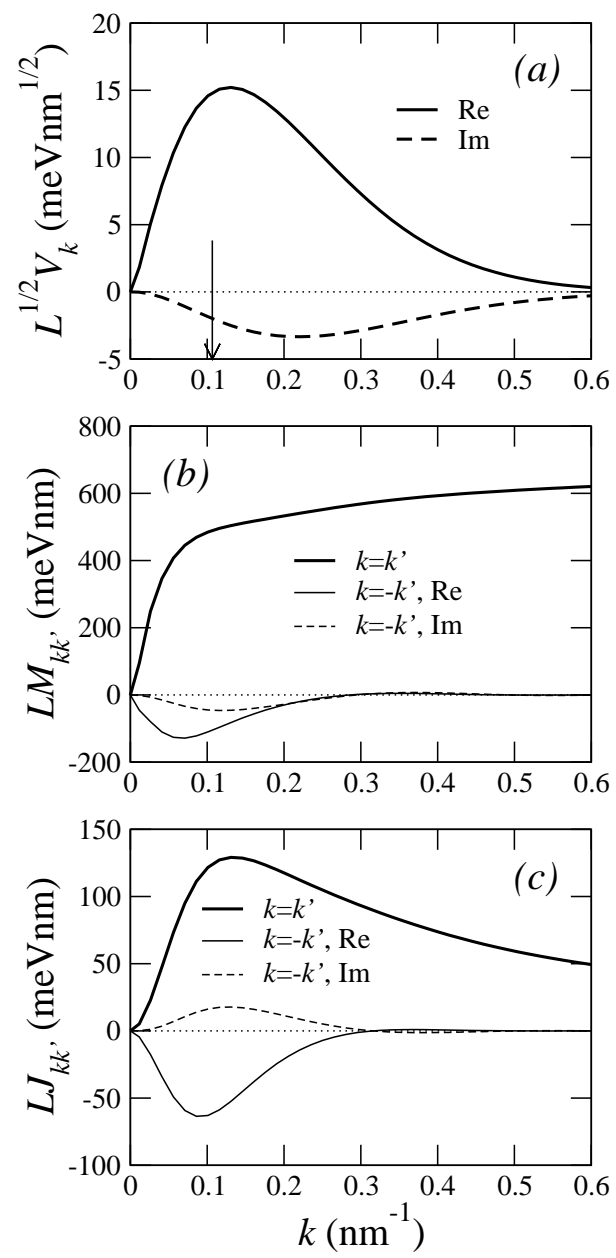

FIG. 13: $k$-dependence of matrix elements of the extended Anderson model. The wire is parameterized with $a_{0}=10 \mathrm{~nm}$, $\xi=0.24, a_{1} / a_{0}=2, V_{0}=0.4 \mathrm{eV}, \kappa=50 \mathrm{~nm}$ and $\gamma=1$. (a) Mixing coupling $V_{k}$. The energy $\epsilon_{d}+U$ is indicated with an arrow. (b, c) Scattering couplings $M_{k k^{\prime}}$ and $J_{k k^{\prime}} . L$ is the length of the wire, where the wave functions are normalized.

scattering solutions of the Hamiltonian Eq. (2.7) are then obtained exactly for two electrons with the boundary condition that for $z \rightarrow \infty$, one electron occupies the lowest bound state, whilst the other is in a forward propagating plane wave state, $\phi_{k}(z) \sim e^{i k z}$. From these solutions we compute the conductance again using the LandauerBüttiker formula.

In Figs. 14 (a,b,c) we compare the results of $\mathcal{T}_{\mathrm{s}}, \mathcal{T}_{\mathrm{t}}$ and conductance $G$ for a wire with the bulge as in Fig. 4. The thin lines are the exact scattering result for two electrons. The solid lines show the exact scattering solutions for the Anderson-type Hamiltonian, for which the matrix elements, and their energy dependence are calculated explicitly. The solution of this Anderson-type model for two electrons, in which the localized level always con- 
tains at least one electron, reproduce the main features of the exact scattering solutions of the original model. The energy dependence of the matrix elements is essential to get this good agreement. Figs. 14 (d,e,f) show the corresponding results for a longer bulge from Fig. 5. Also shown in Fig. 14, dashed lines, are results with the direct exchange term omitted in Eq. (2.7). This term can have a significant quantitative effect, but does not qualitatively change the conductance curves.

We have also solved a similar model in which plane waves, rather than exact scattering states of the noninteracting problem, were used. However, this gave poor agreement with the exact results. We conclude that an Anderson-type model is adequate for a near-perfect quantum wire provided that a suitable basis set is used and the energy-dependence of the matrix elements is accurately determined. Future work will focus on the manyelectron properties of this effective Hamiltonian, including 'Kondo' and 'mixed valence' regimes.
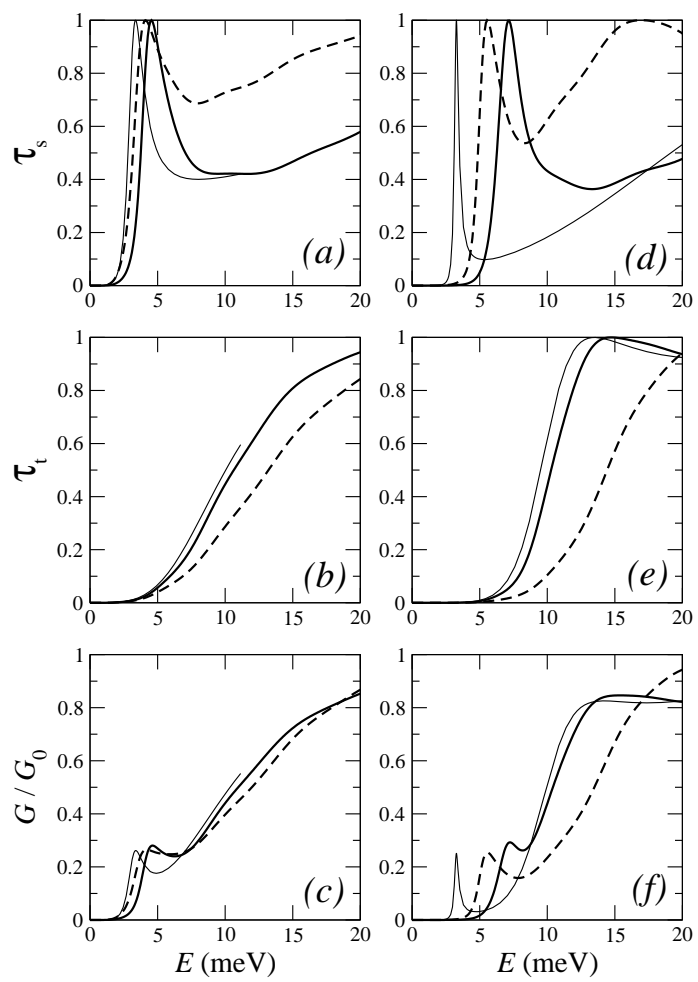

FIG. 14: Singlet (a, d) and triplet (b, e) transmission probabilities and corresponding conductances $(\mathrm{c}, \mathrm{f})$. Parameters for the left set are as in Fig. 13, for the right set: $a_{0}=10 \mathrm{~nm}$, $\xi=0.15, a_{1} / a_{0}=4, V_{0}=0.4 \mathrm{eV}, \kappa=100 \mathrm{~nm}$ and $\gamma=0.9$. Thin lines represent exact results from Eq. (2.1), thick lines are results from Eq. 2.7). Dashed lines show results where the exchange term in Eq. 2.7) is neglected.

\section{F. Approximate methods}

It is not easy to get sufficiently accurate numerical solutions for the case of more than two electrons. Therefore it would be extremely useful if an accurate approximative method could be applied. The simplest approximation (presented here for the case of two electrons) is be the first iteration in solving the Hartree-Fock equations, as we presented in Section III for the case of bound states.

We assume the two-body wave functions consist of a single particle state $\left|\varphi^{(1)}\right\rangle$ and scattering state $|\chi\rangle$ with energy $E$. The two-electron wavefunction has then the form (see Appendix C),

$$
|\psi\rangle=\sum_{i j} \varphi_{i} \chi_{j} c_{i j}^{\left(S, S_{z}\right) \dagger}|0\rangle .
$$

The coefficients $\varphi_{i}$ are known, therefore only coefficients $\chi_{i}$ must be determined. For the singlet state the simplest approximation is obtained if we perform the first iteration of the Hartree-Fock method subject to additional condition that the electron has energy $E$ ("restricted HartreeFock" approximation):

$$
\left\langle 0\left|c_{i} H_{1}\right| \chi\right\rangle+\sum_{j} U_{i j}\left|\varphi_{j}\right|^{2} \chi_{i}=E \chi_{i}
$$

where, using Eq. (2.2), $\left\langle 0\left|c_{i} H_{1}\right| \chi\right\rangle=-t\left(\chi_{i-1}+\chi_{i+1}\right)+$ $\epsilon_{i} \chi_{i}$. This is just the tight binding results for a single electron moving in an effective potential $\epsilon_{i}+\sum_{j} U_{i j}\left|\varphi_{j}\right|^{2}$.

For the triplet state, the result is:

$$
\left\langle 0\left|c_{i} H_{1}\right| \chi\right\rangle+\sum_{j} U_{i j}\left|\varphi_{j}\right|^{2} \chi_{i}-\sum_{j} U_{i j} \varphi_{j}^{*} \varphi_{i} \chi_{j}=E \chi_{j} .
$$

A better approximation for the singlet case starts from the unrestricted Hartree-Fock approximation, where the energy is

$$
\begin{aligned}
& \langle\psi|H| \psi\rangle=\left\langle\varphi\left|H_{1}\right| \varphi\right\rangle\langle\chi \mid \chi\rangle+\left\langle\chi\left|H_{1}\right| \chi\right\rangle\langle\varphi \mid \varphi\rangle+ \\
& \quad+(-1)^{S}\left(\left\langle\varphi\left|H_{1}\right| \chi\right\rangle\langle\chi \mid \varphi\rangle+\left\langle\chi\left|H_{1}\right| \varphi\right\rangle\langle\varphi \mid \chi\rangle\right)+ \\
& \quad+\frac{1}{2} \sum_{i j} U_{i j}\left|\varphi_{i} \chi_{j}+\chi_{i} \varphi_{j}\right|^{2},
\end{aligned}
$$

and the norm is given with

$$
\langle\psi \mid \psi\rangle=\sum_{i}\left|\chi_{i}\right|^{2}+\left|\sum_{i} \varphi_{i}^{(1) *} \chi_{i}\right|^{2} .
$$

The coefficients $\chi_{i}$ are calculated from the Hartree-Fock equations based on the variation principle ("unrestricted Hartree-Fock" approximation),

$$
\begin{gathered}
\left\langle 0\left|c_{i} H_{1}\right| \chi\right\rangle+\sum_{j} U_{i j}\left|\varphi_{j}\right|^{2} \chi_{i}+\sum_{j} U_{i j} \varphi_{j}^{*} \varphi_{i} \chi_{j}+ \\
+\left(E-E_{1}^{(1)}\right) \sum_{j} \varphi_{j}^{*} \varphi_{i} \chi_{j}=E \chi_{i} .
\end{gathered}
$$


In Fig. 15 the effective one dimensional potential in Eq. (4.15) is plotted for $\gamma=0,0.5$ and 1. The shaded region represents the position and the width of single particle resonance in this effective potential. This resonance corresponds to the singlet resonance presented in Fig. 16(a) (dashed line), for wire parameters given in Fig. 1 together with exact result, full line, and calculated from Eq. 4.15).

We also show in Fig. 16.(a) the exact result and the corresponding result for an unrestricted Hartree-Fock scheme for which the wavefunctions of up and down spin electrons are different. As expected, the unrestricted method gives a more accurate result though both methods reproduce the main features, the main discrepancy being an overall energy shift. Similarly in Fig. 16(b) we present the corresponding triplet resonance curve for parameters from Fig. 5. Again, the overall agreement with the exact result is good apart from an overall energy shift.

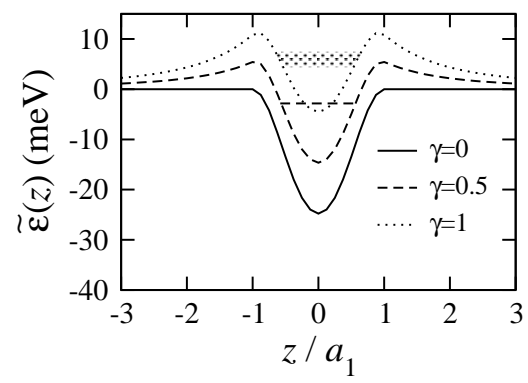

FIG. 15: Effective potential from Eq. (4.15) and for the wire with parameters as in Fig. 1 .
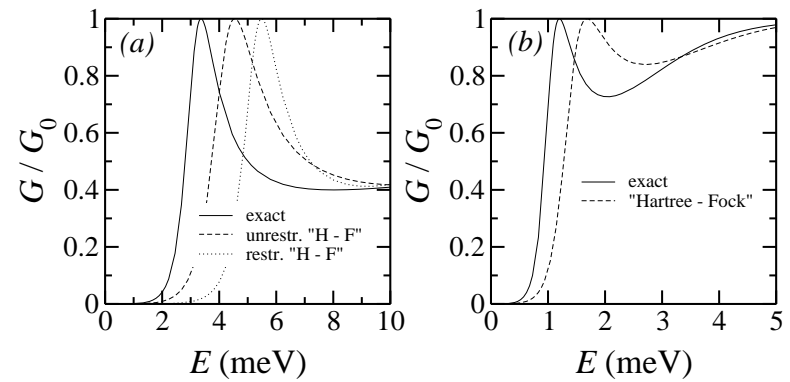

FIG. 16: (a) Singlet resonance (parameters from Fig. 4). Exact result and approximations of Eqns. (4.15) and (4.19) are shown. (b) Triplet resonance (parameters from Fig. 5). Exact result and approximation of Eqn. (4.16) are shown.

\section{SUMMARY AND CONCLUSIONS}

We have shown that quantum wires with weak longitudinal confinement, or open quantum dots, can give rise to spin-dependent, Coulomb blockade resonances when a single electron is bound in the confined region. This is a universal effect in one-dimensional systems with very weak longitudinal confinement. The emergence of a specific structure at $G(E) \sim \frac{1}{4} \frac{2 e^{2}}{h}$ and $G \sim \frac{3}{4} \frac{2 e^{2}}{h}$ is a consequence of the singlet and triplet nature of the resonances and the probability ratio 1:3 for singlet and triplet scattering and as such is a universal effect. A comprehensive numerical investigation of open quantum dots using a wide range of parameters shows that singlet resonances are always at lower energies than the triplets, in accerdance with the corresponding theorem for bound states 38 . With increasing in-plane magnetic field, the resonances shift their position and eventually merge in the conductance plateau at $G \sim e^{2} / h$. With increasing source-drain bias we have shown why the higher triplet resonance weakens at the expense of the singlet, with the latter surviving to the point where the conductance steps themselves disappear.

The existence of the conductance anomalies is a direct consequence of an effective double-barrier potential seen by the conduction electrons propagating from source to drain contacts under the influence of a bound electron. For a symmetric one-electron confining potential, the existence of a bound state is guaranteed but this is not necessarily the case when the confinement is asymmetric. Such asymmetry in the confining potential may be easily achieved under a finite source drain bias and indeed, this was reportef in some of the experiments on gated quantum wires 83 . These experiments show that as the source-drain bias is increased from zero, an anomaly appears at $G \sim 0.25\left(2 e^{2} / h\right)$, coexisting with the $0.7\left(2 e^{2} / h\right)$ anomaly. Eventually, at larger bias, the remaining anomaly also disappears but only when the conductance steps themselves are on the point of disappearing, showing that the singlet anomaly is extremely robust. This behaviour is consistent with our model since under bias the triplet resonant bound-state will eventually disappear because the confining potential in the $x$-direction will only accommodate a single one-electron bound state, giving rise to a singlet resonance only. This is shown schematically in Fig. 17 where we also indicate the surviving singlet becoming broader with increasing bias resulting in a more pronounced step, as observed.

Finally, we speculate on the exciting possibility that these anomalies in conduction are themselves a signature for a new kind of conducting state in ultra clean wires close to the conduction threshold. Indeed, there is some experimental evidence for this in that the anomalies discussed above merge into a conductance step at $e^{2} / h$ under quite moderate magnetic fields and in the cleanest samples this behaviour is sometimes even seen in zero magnetic field. This suggests that there may be an underlying spin polarized state associated with the propagating electrons in the quasi $1 \mathrm{D}$ region. Such a spin-polarized state would appear to violate the Lieb-Mattis theorem 38 and would also need to be made consistent with our above explanation in terms of singlet and triplet resonances. In this respect we emphasize that the above theory must break down at very low electron density in the wire such that the mean separation between electrons in the wire is 

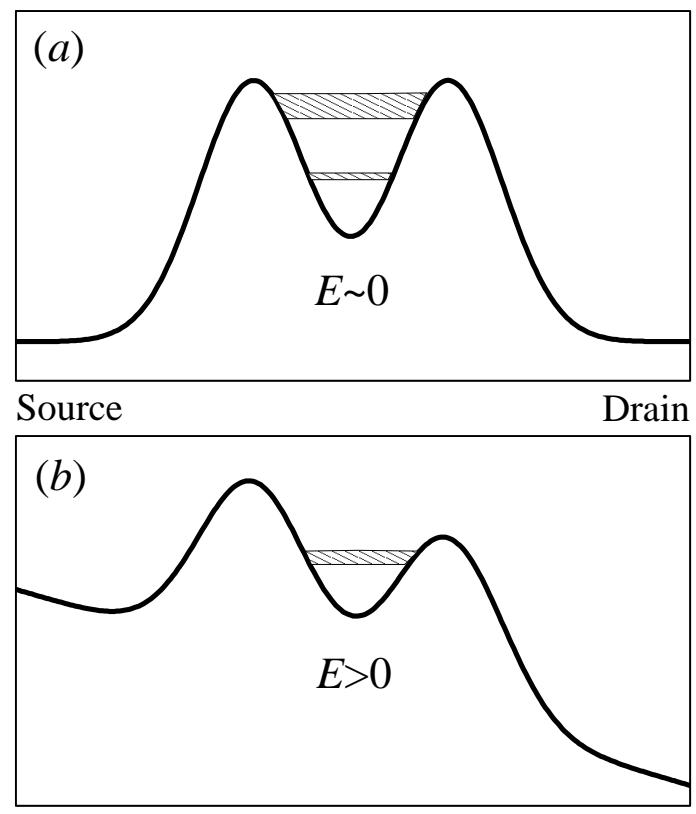

FIG. 17: Effective double barrier showing singlet and triplet resonance with very small source-drain bias (a) and large source-drain bias (b).

somewhat greater than the effective Bohr radius, the socalled strong correlation regime. In practical situations it is very difficult to avoid some kind of weak potential fluctuation which traps one electron. Indeed this may ultimately be impossible since even in a nominally perfect wire, the presence of a single electron will polarise its environment leading to a potential well which will bind the electron giving rise to a Coulomb blockade for the remaining electrons, though the energy scale (temperature) for this may be very low making it susceptible to masking the other effects.

The main question is whether or not this confinement is sufficiently large for the electron density to exceed to the inverse Bohr radius when the wire begins to conduct. If the density remains low at this conductance threshold then we cannot ignore the mutual interaction between all electrons in the wire region, or even treat them selfconsistently. In this situation, a more appropriate picture would be one in which the Coulomb repulsion dominates and maintains roughly equal separation between the electrons as in a Wigner chain. On the other hand, if the mean electron density is of order, or greater,than the inverse Bohr radius, then an open quantum dot picture with effective resonance levels for the propagating electron is more appropriate, as discussed in this paper.

At low temperatures strong many-body effects are indicated fram the activation-like behaviour of the conductance 15 and the thermopower coeficient 16 . As discused in our recend thermopower analysis, Ref. 22, the anomaly at low-temperatures may well be a many-body Kondo-like effect contained within our extended Anderson model, Eq. (2.7), and studied recently in Ref. 27, but not within the two-electron approximation we have used here and in some our earlier papers. It may well be that the two-electron approximation breaks down at low temperatures. The model presented here differs from the standard Anderson model in that the hybridisation term contains the factor $n_{-\sigma}$, and hence disappears when the localized orbital is unoccupied. This reflects the fact that an effective double-barrier structure and resonant bound state occurs via Coulomb repulsion only because of the presence of a localized electron The standard results for the single impurity problem 41 thus cannot be applied directly to this effective model, and are a subject of current reaserch 12 . However, a Kondo-like resonance is expected 27, 43 , for which many-body effects would dominate with a breakdown of our two electron approximation.

\section{APPENDIX A: CYLINDRICAL WIRE}

\section{Single electron basis}

A single electron in the wire considered here is described with the wave function $\Psi(r, \varphi, z)$, which is a solution of the Schrödinger equation

$$
-\frac{\hbar^{2}}{2 m^{*}} \nabla^{2} \Psi(r, \varphi, z)+V(r, z) \Psi(r, \varphi, z)=E \Psi(r, \varphi, z),
$$

where the effects of nonparabolicity are neglected and the effective mass is taken constant, $m^{*}=0.067 m_{\text {elec }}$ with dielectric constant 12.5, appropriate for GaAs36.

At fixed $z$ the wave function $\Psi(r, \varphi, z)$ is expanded in a two-dimensional basis $\Phi_{m n}(r, \varphi ; z)$ for the corresponding potential $V(r ; z)$, Eq. (2.7). The coefficients in such an expansion over channels are $\psi_{m n}(z)$

$$
\Psi(r, \varphi, z)=\sum_{n=0}^{\infty} \sum_{m=-n}^{n} \psi_{m n}(z) \Phi_{m n}(r, \varphi ; z) .
$$

The transverse wavefunftions, $\Phi_{m n}(r, \varphi ; z)$, depend only parametrically on $z$ and take the form:

$$
\Phi_{m n}(r, \varphi ; z)= \begin{cases}A_{m n}(z) J_{m}\left(k_{m n}(z) r\right) e^{i m \varphi} & r<\frac{a(z)}{2} \\ {\left[B_{m n}(z) B_{m}^{(1)}\left(\kappa_{m n}(z) r\right)+\right.} & r>\frac{a(z)}{2} \\ \left.+C_{m n}(z) B_{m}^{(2)}\left(\kappa_{m n}(z) r\right)\right] e^{i m \varphi} & \end{cases}
$$

$$
\begin{gathered}
k_{m n}(z)=\sqrt{\frac{2 m^{*} \epsilon_{m n}(z)}{\hbar^{2}}}, \\
\kappa_{m n}(z)=\sqrt{\frac{2 m^{*}\left|\epsilon_{m n}(z)-V_{0}\right|}{\hbar^{2}}} .
\end{gathered}
$$


Here $B_{m}^{(1)}=I_{m}$ and $B_{m}^{(2)}=K_{m}$ are appropriate Bessel eigenfunctions 34 for $\epsilon_{m n} \leq V_{0}$ with $B_{m}^{(1)}=J_{m}$ and $B_{m}^{(2)}=Y_{m}$, for $\epsilon_{m n}>V_{0}$. The coefficients $A_{m n}, B_{m n}$, $C_{m n}$ and energies $\epsilon_{m n}$ are determined from the boundary conditions and the normalization of wave functions.

Substituting Eq. (A2) into Eq. A1 and integrating over $r$ and $\varphi$ leads to following coupled ordinary differential equations for $\psi_{m n}$,

$$
\begin{aligned}
\psi_{m n}^{\prime \prime} & +\left[k^{2}-k_{m n}^{2}(z)+a_{m n n}(z)\right] \psi_{m n}+\quad(\mathrm{A} 4) \\
& +\sum_{n \neq n^{\prime}} b_{m n n^{\prime}}(z) \psi_{m n^{\prime}}^{\prime}+\sum_{n \neq n^{\prime}} a_{m n n^{\prime}}(z) \psi_{m n^{\prime}}=0
\end{aligned}
$$

where the coupling coefficients are

$$
\begin{aligned}
& a_{m n n^{\prime}}(z)=2 \pi \int_{0}^{R} \Phi_{m n}^{*}(r, \varphi ; z) \frac{\partial^{2}}{\partial z^{2}} \Phi_{m n^{\prime}}(r, \varphi ; z) r \mathrm{~d} r \\
& b_{m n n^{\prime}}(z)=4 \pi \int_{0}^{R} \Phi_{m n}^{*}(r, \varphi ; z) \frac{\partial}{\partial z} \Phi_{m n^{\prime}}(r, \varphi ; z) r \mathrm{~d} r .
\end{aligned}
$$

The coefficients coupling channels with different $m$ are zero due to the orthogonality of $e^{i m \varphi}$ for different $m$.

Note that the Schrödinger equation, Eq. (A1), is invariant under the transformation

$$
\begin{aligned}
\mathbf{r} & \rightarrow \Lambda \mathbf{r} \\
E, V & \rightarrow \Lambda^{-2} E, \Lambda^{-2} V .
\end{aligned}
$$

\section{Extended Hubbard Hamiltonian}

We consider here the case when the variation in wire width is small, resulting in small derivatives of the coefficients in Eqs. A5a, A5b. We consider only electrons with energy below the second channel and hence Eq. (A4) reduces to a single equation for motion in $z$ direction, with the potential

$$
\epsilon(z)=\epsilon_{00}(a(z))+a^{\prime 2}(z) \tilde{\epsilon}_{00}(a(z)) .
$$

The first term is the energy of the first channel and the second is related to $a_{000}(z)$ from Eq. (A4). $a^{\prime}(z)$ is the derivative of the wire diameter with respect to $z$. The second term in Eq. (A8) is always positive since

$$
\tilde{\epsilon}_{00}(a)=\frac{\hbar^{2} \pi}{m^{*}} \int_{0}^{R}\left(\frac{\partial \Phi_{00}(r, \varphi)}{\partial a}\right)^{2} r \mathrm{~d} r .
$$

The potential Eq. (A8) is constant for large $|z|$ and set to zero for convenience, i.e.

$$
\epsilon(z) \rightarrow \epsilon(z)-\epsilon(\infty)
$$

In Fig. 18(a) $\epsilon_{00}(a)$ and $\tilde{\epsilon}_{00}(a)$ are presented as a function of wire diameter. Figs. 18 (b) and (c) show the variation of one-dimensional potential $\epsilon(z)$ along the wire.
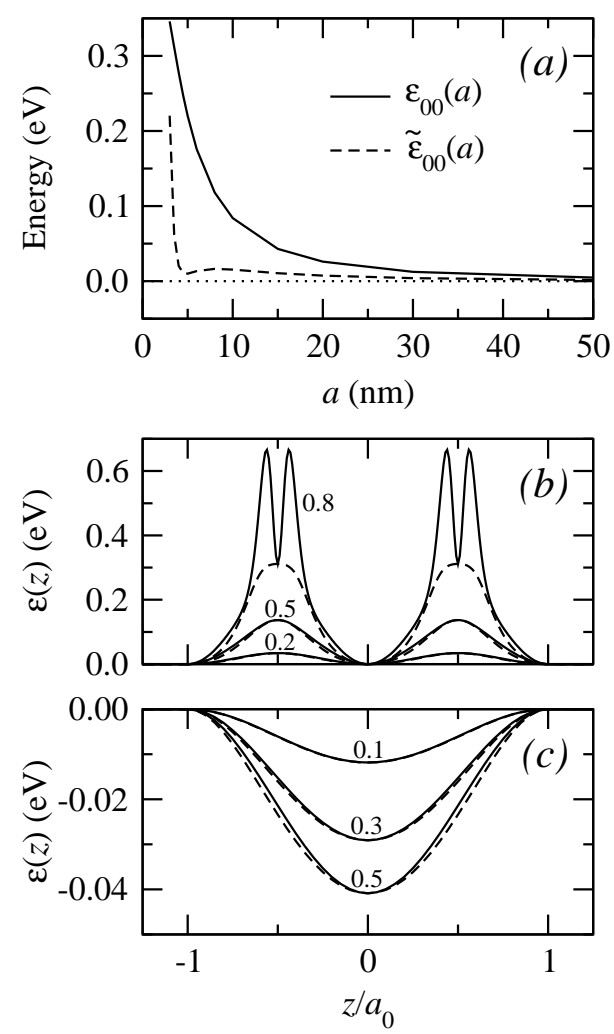

FIG. 18: (a) Dependence of $\epsilon_{00}$ and $\tilde{\epsilon}_{00}$ in Eq. (A\&) on wire diameter for $V_{0}=0.4 \mathrm{eV}$. (b) One dimensional potential Eq. (A\&) for the wire shape Eq. (2.8a) and various values of $\xi$. Dashed lines correspond to the contribution $\epsilon_{00}$. (c) The same as in (b) but for wire shape Eq. (2.8b).

The single-electron Hamiltonian in the single channel approximation then becomes

$$
H_{1}=-\frac{\hbar^{2}}{2 m^{*}} \frac{\mathrm{d}^{2}}{\mathrm{~d} z^{2}}+\epsilon(z) .
$$

This is readily generalized to many electrons,

$$
\begin{aligned}
& H=\sum_{\sigma} \int \psi_{\sigma}^{\dagger}(z)\left[-\frac{\hbar^{2}}{2 m^{*}} \frac{\mathrm{d}^{2}}{\mathrm{~d} z^{2}}\right] \psi_{\sigma}(z) \mathrm{d} z+\quad(\mathrm{A} 12) \\
& +\frac{1}{2} \sum_{\sigma, \sigma^{\prime}} \iint \psi_{\sigma}^{\dagger}(z) \psi_{\sigma^{\prime}}^{\dagger}\left(z^{\prime}\right) U\left(z, z^{\prime}\right) \psi_{\sigma^{\prime}}\left(z^{\prime}\right) \psi_{\sigma}(z) \mathrm{d} z \mathrm{~d} z^{\prime},
\end{aligned}
$$

where $\psi_{\sigma}^{\dagger}(z)$ creates an electron with spin $\sigma$ at coordinate $z$ and

$$
U\left(z_{i}, z_{j}\right)=\frac{e^{2}}{4 \pi \epsilon \epsilon_{0} d\left(z_{i}, z_{j}\right)}
$$


with

$$
\frac{1}{d\left(z_{i}, z_{j}\right)}=\int \mathrm{d} \mathbf{r}_{i} \mathrm{~d} \mathbf{r}_{j} \frac{\left|\Phi_{00}\left(\mathbf{r}_{i} ; z_{i}\right)\right|^{2}\left|\Phi_{00}\left(\mathbf{r}_{j} ; z_{j}\right)\right|^{2}}{\sqrt{\left(z_{i}-z_{j}\right)^{2}+\left|\mathbf{r}_{i}-\mathbf{r}_{j}\right|^{2}}}
$$

The Hamiltonian is further discretized at points $z_{j}=j \Delta$, new creation operators are defined as

$$
c_{j \sigma}^{\dagger}=\sqrt{\Delta} \psi_{\sigma}^{\dagger}\left(z_{j}\right) .
$$

For sufficiently small $\Delta$ the difference formula is justified,

$$
\left[\frac{\mathrm{d}^{2}}{\mathrm{~d} z^{2}} \psi_{\sigma}(z)\right]_{z=z_{i}} \approx \frac{\psi_{\sigma}\left(z_{i-1}\right)-2 \psi_{\sigma}\left(z_{i}\right)+\psi_{\sigma}\left(z_{i+1}\right)}{2 \Delta^{2}},
$$

and Eq. (A13) becomes the discretized extended Hubbard Hamiltonian,

$$
H=\sum_{\sigma} H_{1 \sigma}+\frac{1}{2} \sum_{i \neq j} U_{i j} n_{i} n_{j}+\sum_{i} U_{i j} n_{i \uparrow} n_{j \downarrow},
$$

where $H_{1 \sigma}$ is single-particle contribution for spin $\sigma$,

$$
H_{1 \sigma}=-t \sum_{i}\left(c_{i+1 \sigma}^{\dagger} c_{i \sigma}+c_{i \sigma}^{\dagger} c_{i+1 \sigma}\right)+\sum_{i} \epsilon_{i} n_{i \sigma}
$$

with $n_{i \sigma}=c_{i \sigma}^{\dagger} c_{i \sigma}, n_{i}=\sum_{\sigma} n_{i \sigma}$, hoping parameter,

$$
t=\frac{\hbar^{2}}{2 m^{*} \Delta^{2}}
$$

and $\epsilon_{i}=2 t+\epsilon\left(z_{i}\right)$. The effective distance between electrons at $z_{i}$ and $z_{j}$ is after integrating Eq. (A13) over angular variables,

$$
\begin{gathered}
\frac{1}{d\left(z_{i}, z_{j}\right)}=8 \pi \int_{0}^{R} r_{i} \mathrm{~d} r_{i} \int_{0}^{R} r_{j} \mathrm{~d} r_{j}\left|\Phi_{00}\left(r_{i} ; z_{i}\right)\right|^{2} \times \\
\quad \times\left|\Phi\left(r_{j} ; z_{j}\right)\right|^{2} \frac{\mathrm{K}\left(-\frac{4 r_{i} r_{j}}{\left(z_{i}-z_{j}\right)^{2}+\left(r_{i}-r_{j}\right)^{2}}\right)}{\sqrt{\left(z_{i}-z_{j}\right)^{2}+\left(r_{i}-r_{j}\right)^{2}}}, \quad
\end{gathered}
$$

where $\mathrm{K}$ is the complete elliptic integral of the first kind. $d\left(z_{i}, z_{j}\right)$ can be decomposed into distance along the wire and effective distance in the lateral direction, $\lambda\left(z_{i}, z_{j}\right) a_{0}$, i.e.

$$
\frac{1}{d\left(z_{i}, z_{j}\right)}=\frac{1}{\sqrt{\left(z_{i}-z_{j}\right)^{2}+\left[\lambda\left(z_{i}, z_{j}\right) a_{0}\right]^{2}}} .
$$

The distance $\lambda\left(z_{i}, z_{j}\right)$ is invariant under the transformation Eq. (A6), and hence the potential, Eq. (A13), transforms as

$$
U \rightarrow \Lambda^{-1} U
$$

For convenience we also take into account possible screening with screening length $\kappa$, i.e.

$$
U_{i j} \rightarrow U_{i j} e^{-\frac{\left|z_{i}-z_{j}\right|}{\kappa}}
$$

Under the transformation Eq. (A22) the screening length should be multiplied by $\Lambda$. In Fig. 19 the parameter $\lambda$ is plotted for some typical cases, showing its dependence on wire width.
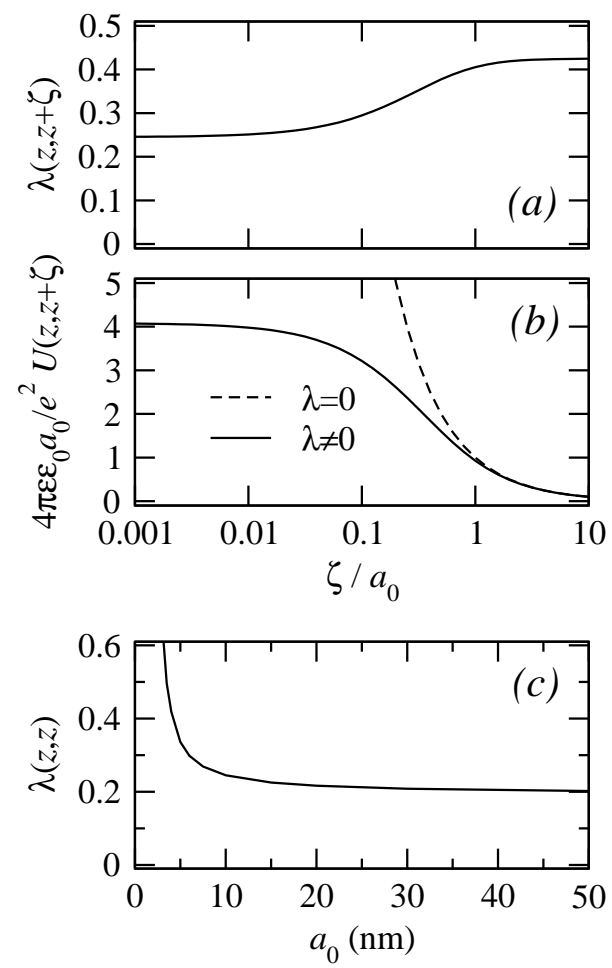

FIG. 19: (a) Lateral distance vs. separation along the wire. (b) Electron - electron interaction as a function of longitudinal separation. In both cases is $a_{0}=10 \mathrm{~nm}$ and $V_{0}=0.4 \mathrm{eV}$ and $a=$ const. (c) Lateral distance at fixed $z$ vs. wire diameter at $V_{0}=0.4 \mathrm{eV}$.

\section{APPENDIX B: TWO-ELECTRON WAVE FUNCTIONS}

Wave function for the case of two electrons are expressed in terms of a set of operators $c_{i j}^{\left(S, S_{z}\right) \dagger}$ creating an electron pair at sites $i$ and $j$ with spin $S$ and $z$-component $S_{z}$, i.e.

$$
|\psi\rangle=\sum_{i j} \psi_{i j} c_{i j}^{\left(S, S_{z}\right) \dagger}|0\rangle
$$

The base states 


$$
\begin{aligned}
c_{i j}^{(0,0) \dagger}|0\rangle & =\frac{c_{i \uparrow}^{\dagger} c_{j \downarrow}^{\dagger}-c_{i \downarrow}^{\dagger} c_{j \uparrow}^{\dagger}}{\sqrt{2}}|0\rangle, \\
c_{i j}^{(1,1) \dagger}|0\rangle & =c_{i \uparrow}^{\dagger} c_{j \uparrow}^{\dagger}|0\rangle, \\
c_{i j}^{(1,0) \dagger}|0\rangle & =\frac{c_{i \uparrow}^{\dagger} c_{j \downarrow}^{\dagger}+c_{i \downarrow}^{\dagger} c_{j \uparrow}^{\dagger}}{\sqrt{2}}|0\rangle, \\
c_{i j}^{(1,-1) \dagger}|0\rangle & =c_{i \downarrow}^{\dagger} c_{j \downarrow}^{\dagger}|0\rangle .
\end{aligned}
$$

form a complete set.

If $|\psi\rangle$ is a solution of Schrödinger equation

$$
H|\psi\rangle=E|\psi\rangle,
$$

then the coefficients $\widetilde{\psi}_{i j}$ solve the system of linear equations

$$
\begin{gathered}
t\left(\widetilde{\psi}_{i-1 j}+\widetilde{\psi}_{i+1 j}+\widetilde{\psi}_{i j-1}+\widetilde{\psi}_{i j+1}\right)= \\
=\left(\epsilon_{i}+\epsilon_{j}+U_{i j}-E\right) \widetilde{\psi}_{i j},
\end{gathered}
$$

where we use compact notation

$$
\widetilde{\psi}_{i j}=\frac{1}{\sqrt{2}}\left(\psi_{i j}+(-1)^{S} \psi_{j i}\right) .
$$

In the basis Eq. (B1) the number of electrons on site $i$ is

$$
\left\langle\psi\left|n_{i}\right| \psi\right\rangle=2 \sum_{j}\left|\widetilde{\psi}_{i j}\right|^{2}
$$

the current for sites $i$ and $i+1$ is

$$
\left\langle\psi\left|j^{i, i+1}\right| \psi\right\rangle=-\frac{4 t \Delta}{\hbar} \operatorname{Im} \sum_{j} \widetilde{\psi}_{i j}^{*} \widetilde{\psi}_{i+1 j},
$$

the energy is

$$
\begin{aligned}
& \langle\psi|H| \psi\rangle= \\
& \quad-t \sum_{i j} \widetilde{\psi}_{i j}^{*}\left(\widetilde{\psi}_{i+1 j}+\widetilde{\psi}_{i-1 j}+\widetilde{\psi}_{i j+1}+\widetilde{\psi}_{i j-1}\right)+ \\
& \quad+\sum_{i j}\left(\epsilon_{i}+\epsilon_{j}+U_{i j}\right)\left|\widetilde{\psi}_{i j}\right|^{2}
\end{aligned}
$$

and the norm of the wave function Eq. (B1) is given with

$$
\langle\psi \mid \psi\rangle=\sum_{i j}\left|\widetilde{\psi}_{i j}\right|^{2}
$$

We consider quantum wires which are almost perfect but for which there is a very weak effective potential, giving rise to bound states. The cross-sections of these wires are sufficiently small that the lowest transverse channel approximation is adequate for the energy range of interest. The smooth variation in cross-section also guarantees that inter-channel mixing is negligible. We study here only wires with one weak bulge around $z=0$. There should exist single particle bound states of the system and $E_{1}^{(\alpha)}$ is the energy of the state $\alpha$. The energy of two electron states is shifted and defined to be zero, if one electron is bound and the other at the bottom of the single electron band, i.e.

$$
E \rightarrow E-E_{1}^{(1)} .
$$

With this definition, the energy of two bound electrons is negative whereas it is positive when only one electron is bound.

\section{APPENDIX C: HARTREE-FOCK APPROXIMATION}

Here we neglect the Coulomb interaction between electrons $(\gamma=0)$. In the ground state both electrons are in the same state $|\varphi\rangle$ and the singlet wavefunction is

$$
|\psi\rangle=\frac{1}{\sqrt{2}} \sum_{i j} \varphi_{i} \varphi_{j} c_{i j}^{(0,0) \dagger}|0\rangle .
$$

For finite $\gamma$ the best one-electron wavefunctions, $\varphi_{i}$, are determined by minimizing the energy,

$$
\frac{\partial}{\partial \varphi_{i}^{*}} \frac{\langle\psi|H| \psi\rangle}{\langle\psi \mid \psi\rangle}=0 .
$$

Which leads to the equation

$$
\frac{\partial}{\partial \varphi_{i}^{*}}\langle\psi|H| \psi\rangle-\left(E+E_{1}^{(1)}\right) \frac{\partial}{\partial \varphi_{i}^{*}}\langle\psi \mid \psi\rangle=0,
$$

where we have set

$$
\frac{\langle\psi|H| \psi\rangle}{\langle\psi \mid \psi\rangle}=E+E_{1}^{(1)},
$$

taking into account the energy shift Eq. (B10). The expectation value of energy and the norm is

$$
\langle\psi|H| \psi\rangle=2\left\langle\varphi\left|H_{1}\right| \varphi\right\rangle\langle\varphi \mid \varphi\rangle+\sum_{i j} U_{i j}\left|\varphi_{i}\right|^{2}\left|\varphi_{j}\right|^{2},
$$

and

$$
\langle\psi \mid \psi\rangle=\langle\varphi \mid \varphi\rangle^{2} .
$$

From Eq. (C3) follows a system of equations for coefficients $\varphi_{i}$

$$
\left\langle 0\left|c_{i} H_{1}\right| \varphi\right\rangle+\sum_{j} U_{i j}\left|\varphi_{j}\right|^{2} \varphi_{i}=E_{\mathrm{hf}} \varphi_{i},
$$

where $\left\langle 0\left|c_{i} H_{1}\right| \varphi\right\rangle=-t\left(\varphi_{i-1}+\varphi_{i+i}\right)+\epsilon_{i} \varphi$ is a oneelectron tight-binding Hamiltonian, $\sum_{j} U_{i j}\left|\varphi_{j}\right|^{2}$ is a Hartree potential and

$$
E_{\mathrm{hf}}=E+E_{1}^{(1)}-\frac{\left\langle\varphi\left|H_{1}\right| \varphi\right\rangle}{\langle\varphi \mid \varphi\rangle}
$$


is the so-called Hartree-Fock energy. The energy of a bound state is then given by

$$
E=2 E_{\mathrm{hf}}-\sum_{i j} U_{i j}\left|\varphi_{i}\right|^{2}\left|\varphi_{j}\right|^{2}-E_{1}^{(1)}
$$

where, due to double-counting of the interactions in single electron energies, $E_{\mathrm{hf}}$ is subtracted. In Fig. 20 electron density for singlet states with different $\gamma$ is presented for a shorter bulge with parameters as in Fig. 4 .

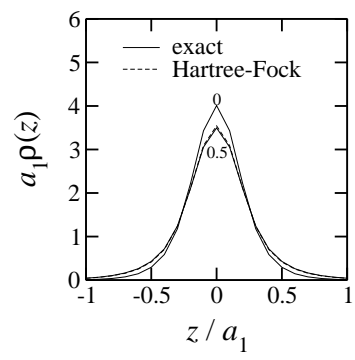

FIG. 20: Electron density for singlet states with different $\gamma$. Full line corresponds to exact results and dashed line to the Hartree-Fock approximation. Parameters as in Fig. 14.

In the case of triplet two-electron states, the single electron states are different, $|\varphi\rangle$ and $|\bar{\varphi}\rangle$. Choosing these to be orthogonal, we get

$$
|\psi\rangle=\sum_{i j} \varphi_{i} \bar{\varphi}_{j} c_{i j}^{(1,1) \dagger}|0\rangle
$$

The energy is now

$$
\begin{aligned}
\langle\psi|H| \psi\rangle & =\left\langle\varphi\left|H_{1}\right| \varphi\right\rangle\langle\bar{\varphi} \mid \bar{\varphi}\rangle \\
& +\left\langle\bar{\varphi}\left|H_{1}\right| \bar{\varphi}\right\rangle\langle\varphi \mid \varphi\rangle+\frac{1}{2} \sum_{i j} U_{i j}\left|\varphi_{i} \bar{\varphi}_{j}-\bar{\varphi}_{i} \varphi_{j}\right|^{2},
\end{aligned}
$$

and the norm is

$$
\langle\psi \mid \psi\rangle=\langle\varphi \mid \varphi\rangle\langle\bar{\varphi} \mid \bar{\varphi}\rangle
$$

The system of equations for the coefficients $\varphi_{i}$ (and equivalent for $\bar{\varphi}_{i}$ ) is

$$
\left\langle 0\left|c_{i} H_{1}\right| \varphi_{i}\right\rangle+\sum_{j} U_{i j}\left|\bar{\varphi}_{j}\right|^{2} \varphi_{i}-\sum_{j} U_{i j} \bar{\varphi}_{j}^{*} \bar{\varphi}_{i} \varphi_{j}=E_{\mathrm{hf}} \varphi_{i},
$$

where

$$
E_{\mathrm{hf}}=E+E_{1}^{(1)}-\frac{\left\langle\bar{\varphi}\left|H_{1}\right| \bar{\varphi}\right\rangle}{\langle\bar{\varphi} \mid \bar{\varphi}\rangle},
$$

Eq. (C13) is a single-particle tight-binding Schödinger equation with Hamiltonian

$$
\tilde{H}_{1}=-\sum_{i} \tilde{t}_{i j}\left(c_{i}^{\dagger} c_{j}+c_{j}^{\dagger} c_{i}\right)+\sum_{i} \tilde{\epsilon}_{i} c_{i}^{\dagger} c_{i}
$$

with potential

$$
\tilde{\epsilon}_{i}=\epsilon_{i}+\sum_{j} U_{i j}\left|\bar{\varphi}_{j}\right|^{2}
$$

and renormalized hoping parameters

$$
\tilde{t}_{i j}=t \delta_{j, i \pm 1}+\sum_{j} U_{i j} \bar{\varphi}_{j}^{*} \bar{\varphi}_{i}
$$

The energy of the triplet bound state is then

$$
E=E_{\mathrm{hf}}+\bar{E}_{\mathrm{hf}}-\frac{1}{2} \sum_{i j} U_{i j}\left|\varphi_{i} \bar{\varphi}_{j}-\bar{\varphi}_{i} \varphi_{j}\right|^{2}-E_{1}^{(1)} .
$$

In Fig. 21 the electron density for singlet (a) and for triplet (b) states are shown for different $\gamma$. Other parameters are taken as in the case of the longer bulge, Fig. 5 . As discussed in the text relating to Fig. 5, Hartree-Fock approximation for the singlet is less reliable since the Coulomb repulsion is stronger due to both electrons being in the same state. Indeed, for $\gamma \sim 0.7$ the HartreeFock approximation does not yield the bound state found in the exact result.
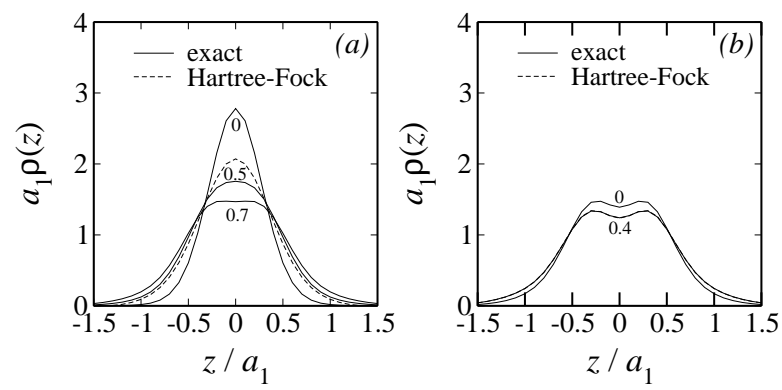

FIG. 21: Electron singlet (a) and triplet (b) state density for various $\gamma$. Parameters are as in Fig. 5 .

\section{ACKNOWLEDGMENTS}

The authors wish to acknowledge N.J. Appleyard, A.V. Khaetskii, C.J. Lambert, M. Pepper and K.J. Thomas for helpful discussions. This work was supported by the EU and the MoD. 
al., Semicond. Sci. Tech. 9, 1939 (1994); R. Rinaldi et al., Phys. Rev. Lett. 73, 2899 (1994).

2 P. Ramvall et al., Appl. Phys. Lett. 71, 918 (1997).

3 A. Yacoby et al., Phys. Rev. Lett. 77, 4612 (1996).

4 A. Kristensen et al., Contributed paper for ICPS24, Jerusalem, August 2-7, 1998.

5 B.J. van Wees et al., Phys. Rev. Lett. 60, 848 (1998).

6 D.A. Wharam et al., J. Phys. C: Solid state Phys. 21, L209 (1988).

7 H. van Houten, C.W.J. Beenakker, and B.J. van Wees, in Semiconductors and Semimetals, Vol. 35, edited by M.A. Reed (Academic Press, New York, 1992).

8 K.J. Thomas et al., Phys. Rev. Lett. 77, 135 (1996); Phys. Rev. B 58, 4846 (1998); Phys. Rev. B 59, 12252 (1999).

9 C.-T. Liang et al., Phys. Rev. B 60, 10687 (1999).

${ }^{10}$ K.S. Pyshkin et al., Phys. Rev. B 62, 15842 (2000).

11 D. Kaufman et al., Phys. Rev. B 59, R10433 (1999).

12 S. Nuttinck et al., Jpn. J. Appl. Phys. 39, 655 (2000); K. Hashimoto at al., Jpn. J. Appl. Phys. 40, 3000 (2001).

13 A. Kristensen et al., Phys. Rev. B 62, 10950 (2000).

14 C.-T. Liang et al., Phys. Rev. B 61, 9952 (2000).

15 C.M. Cronenwett et al., Phys. Rev. Lett. 88, 226805 (2002)

16 N.J. Appleyard et al., Phys. Rev. B 62, R16275 (2000).

17 D.L. Maslov, Phys. Rev. B 52, R14368, 1995.

18 Chuan-Kui Wang and K.-F. Berggren, Phys. Rev. B 57, 4552 (1998).

19 G. Fasol and H. Sakaki, Jpn. J. Appl. Phys. 33, 879 (1994).

20 T. Rejec, A. Ramšak, and J.H. Jefferson, J. Phys., Condens. Matter 12, L233 (2000).

21 T. Rejec, A. Ramšak, and J.H. Jefferson, Phys. Rev. B 62, 12985 (2000).

22 T. Rejec, A. Ramšak, and J.H. Jefferson, Phys. Rev. B 65, 235301 (2002).

23 V.V. Flambaum and M.Yu. Kuchiev, Phys. Rev. B 61, R7869 (2000).

${ }^{24}$ H. Bruus, V. Cheianov, and K. Flensberg, Physica E 10, 97 (2001).
25 O.P. Sushkov, Phys. Rev. B 64, 155319 (2001)

26 Y. Tokura and A. Khaetskii, Physica E 12, 711 (2002).

27 Y. Meir, K. Hirose, and N.S. Wingreen, cond-mat/0207044.

28 U. Meirav et al., Z. Phys. B 85, 357 (1991).

29 T. Rejec, A. Ramšak, and J.H. Jefferson, in Kondo Effect and Dephasing in Low-Dimensional Metallic Systems, edited by V. Chandrasekhar, C. Van Haesendonck, and A. Zawadowski, NATO ARW, Ser. II, Vol. 50 (Kluwer, Dordrecht, 2001).

30 P.W. Anderson, Phys. Rev. 124, 41 (1961); G.D. Mahan, Many-Particle Physics, Plenum Press, New York (1990).

31 K. Jauregui, W. Häusler, and B. Kramer, Euriphys. Lett. 24581 (1993); D.L.J. Tipton, PhD Thesis, King's College London (2001).

32 L.D. Landau and E.M. Lifshitz, Quantum Mechanics (Pergamon Press, Oxford, 1977).

33 J.R. Oppenheimer, Phys. Rev. 32, 361 (1928); N.F. Mott, Proc. Roy. Soc. A 126, 259 (1930).

34 M. Abramowitz and I.A. Stegun, Handbook of mathematical functions, (Dover publications, Inc., New York).

35 R. Landauer, IBM J. Res. Dev. 1, 223 (1957); 32, 306 (1988); M. Büttiker, Phys. Rev. Lett. 57, 1761 (1986).

36 A. Ramšak, T. Rejec, and J.H. Jefferson, Phys. Rev. B 58, 4014 (1998).

37 N.K. Patel et al., Phys. Rev. B 44, 13549 (1991); K.J. Thomas et al., Phil. Mag. B 77, 1213 (1998).

38 E. Lieb and D. Mattis, Phys. Rev. 125, 164 (1962).

39 L.I. Glazman and M.E. Raikh, JETP Lett. 47, 452 (1988); T.K. Ng and P.A. Lee, Phys. Rev. Lett 61, 1768 (1988).

40 C.E. Creffield, J.H. Jefferson, Sarben Sarkar, and D.L.J. Tipton, Phys. Rev. B 62, 7249 (2000).

41 see, e.g., A.C. Hewson, The Kondo problem to heavy fermions, (Cambridge university press, Cambridge, 1997).

42 T. Rejec and A. Ramšak, unpublished.

43 D. Boese and R. Fazio, Europhys. Lett. 56, 576 (2001). 